\title{
UNDECIDABLE FIRST-ORDER THEORIES OF AFFINE GEOMETRIES *
}

\author{
ANTTI KUUSISTO $^{a}$, JEREMY MEYERS $^{b}$, AND JONNI VIRTEMA $^{c}$ \\ ${ }^{a}$ University of Wrocław \\ e-mail address: antti.j.kuusisto@gmail.com \\ ${ }^{b}$ Stanford University \\ e-mail address: jjmeyers@stanford.edu \\ ${ }^{c}$ University of Tampere \\ e-mail address: jonni.virtema@uta.fi
}

\begin{abstract}
Tarski initiated a logic-based approach to formal geometry that studies firstorder structures with a ternary betweenness relation $(\beta)$ and a quaternary equidistance relation $(\equiv)$. Tarski established, inter alia, that the first-order $(\mathrm{FO})$ theory of $\left(\mathbb{R}^{2}, \beta, \equiv\right)$ is decidable. Aiello and van Benthem (2002) conjectured that the FO-theory of expansions of $\left(\mathbb{R}^{2}, \beta\right)$ with unary predicates is decidable. We refute this conjecture by showing that for all $n \geq 2$, the FO-theory of the class of expansions of $\left(\mathbb{R}^{n}, \beta\right)$ with just one unary predicate is already $\Pi_{1}^{1}$-hard and therefore not even arithmetical. We also define a natural and comprehensive class $\mathcal{C}$ of geometric structures $(T, \beta)$, where $T \subseteq \mathbb{R}^{n}$, and show that for each structure $(T, \beta) \in \mathcal{C}$, the FO-theory of the class of expansions of $(T, \beta)$ with a single unary predicate is undecidable. We then consider classes of expansions of structures $(T, \beta)$ with a restricted unary predicate, for example a finite predicate, and establish a variety of related undecidability results. In addition to decidability questions, we briefly study the expressivities of universal MSO and weak universal MSO over expansions of $\left(\mathbb{R}^{n}, \beta\right)$. While the logics are incomparable in general, over expansions of $\left(\mathbb{R}^{n}, \beta\right)$, formulae of weak universal MSO translate into equivalent formulae of universal MSO.
\end{abstract}

\section{INTRODUCTION}

Decidability of theories of structures and classes of structures is a central topic in various different fields of computer science and mathematics, with different motivations and objectives depending on the field in question. In this article we investigate formal theories of geometry in the framework introduced by Tarski [28, 29]. The logic-based framework was originally presented in a series of lectures given in Warsaw in the 1920's. The system is based on first-order structures with two predicates: a ternary betweenness relation $\beta$ and a quaternary equidistance relation $\equiv$. Within this system, $\beta(u, v, w)$ is interpreted to mean that the point

2012 ACM CCS: [Theory of computation]: Models of computation-Computability; Computational complexity and cryptography - Complexity classes; [Mathematics of computing]: Continuous mathematics - Topology - Geometric topology.

* This work was partially supported by grant 129761 of the Academy of Finland. 
$v$ is between the points $u$ and $w$, while $x y \equiv u v$ means that the distance from $x$ to $y$ is equal to the distance from $u$ to $v$. The betweenness relation $\beta$ can be considered to simulate the action of a ruler, while the equidistance relation $\equiv$ simulates the action of a compass. See [27] and [29] for further information about the history and development of Tarski's geometry.

Tarski established in [28] that the first-order theory of $\left(\mathbb{R}^{2}, \beta, \equiv\right)$ is decidable. In [3], Aiello and van Benthem pose the question: "What is the complete monadic $\Pi_{1}^{1}$ theory of the affine real plane?" By affine real plane, the authors refer to the structure $\left(\mathbb{R}^{2}, \beta\right)$. The monadic $\Pi_{1}^{1}$-theory of $\left(\mathbb{R}^{2}, \beta\right)$ is of course essentially the same as the first-order theory of the class of expansions $\left(\mathbb{R}^{2}, \beta,\left(P_{i}\right)_{i \in \mathbb{N}}\right)$ of the the affine real plane $\left(\mathbb{R}^{2}, \beta\right)$ by unary predicates $P_{i} \subseteq \mathbb{R}^{2}$. Aiello and van Benthem conjecture that the theory is decidable. Expansions of $\left(\mathbb{R}^{2}, \beta\right)$ with unary predicates are especially relevant in investigations related to the geometric structure $\left(\mathbb{R}^{2}, \beta\right)$, since in this context unary predicates correspond to regions of the plane $\mathbb{R}^{2}$.

In this article we study structures of the type of $(T, \beta)$, where $T \subseteq \mathbb{R}^{n}$ and $\beta$ is the canonical Euclidean betweenness predicate restricted to $T$, see Section 2.3 for the formal definition. Let $E((T, \beta))$ denote the class of expansions $(T, \beta, P)$ of $(T, \beta)$ with a single unary predicate. The class $E((T, \beta))$ is called the unary expansion class of $(T, \beta)$. We identify a significant collection of canonical structures $(T, \beta)$ with an undecidable first-order theory of $E((T, \beta))$. Informally, if there exists a flat two-dimensional region $R \subseteq \mathbb{R}^{n}$, no matter how small, such that $T \cap R$ is in a certain sense sufficiently dense with respect to $R$, then the first-order theory of $E((T, \beta))$ is undecidable. If the related density conditions are satisfied, we say that $T$ extends linearly in $2 \mathrm{D}$, see Section 2.3 for the formal definition. We prove that for any $T \subseteq \mathbb{R}^{n}$, if $T$ extends linearly in $2 \mathrm{D}$, then the FO-theory of the unary expansion class of $(T, \beta)$ is $\Sigma_{1}^{0}$-hard. We also obtain a partial converse to this result. We observe that $T$ extending linearly in $1 \mathrm{D}$ (see Section 2.3 for the definition) is not a sufficient condition for undecidability of the FO-theory of $E((T, \beta))$.

In addition, we establish that for all $n \geq 2$, the first-order theory of the unary expansion class of $\left(\mathbb{R}^{n}, \beta\right)$ is $\Pi_{1}^{1}$-hard, and therefore not even arithmetical. We thereby refute the conjecture of Aiello and van Benthem from [3]. The results are ultimately based on tiling arguments. The result establishing $\Pi_{1}^{1}$-hardness relies on the recurrent tiling problem of Harel [15] - once again demonstrating the usefulness of Harel's methods.

Our results establish undecidability for a wide range of unary expansion classes of natural geometric structures $(T, \beta)$. In addition to $\left(\mathbb{R}^{2}, \beta\right)$, such structures include for example the rational plane $\left(\mathbb{Q}^{2}, \beta\right)$, the real unit cube $\left([0,1]^{3}, \beta\right)$, and the plane of algebraic reals $\left(\mathbb{A}^{2}, \beta\right)$ - to name a few.

In addition to investigating expansion classes of the type $E((T, \beta))$, we also study expansion classes with a restricted unary predicate. Let $n$ be a positive integer and let $T \subseteq \mathbb{R}^{n}$. Let $F((T, \beta))$ denote the class of structures $(T, \beta, P)$, where the set $P$ is a finite subset of $T$. We establish that if $T$ extends linearly in $2 \mathrm{D}$, then the first-order theory of $F((T, \beta))$ is undecidable. We obtain a $\Pi_{1}^{0}$-hardness result by an argument based on the periodic torus tiling problem of Gurevich and Koryakov [13]. The torus tiling argument can easily be adapted to deal with various different kinds of natural restricted expansion classes of geometric structures $(T, \beta)$. These include classes with a unary predicate denoting - to name a few examples - a polygon, a finite union of closed rectangles, and a semialgebraic set (see [8] for the definition). 
Our results could turn out useful in investigations concerning logical aspects of spatial databases. There is a canonical correspondence between $\left(\mathbb{R}^{2}, \beta\right)$ and $(\mathbb{R}, 0,1, \cdot,+,<)$, see 14 . for example. See the survey [18] for further details on logical aspects of spatial databases.

The betweenness predicate is also studied in spatial logic [2]. The recent years have witnessed a significant increase in the research on spatially motivated logics. Several interesting systems with varying motivations have been investigated, see for example the articles [3, 4, 5, 16, 17, 21, 26, 30, 31]. See also the surveys [1] and [6] in the Handbook of Spatial Logics [2], and the Ph.D. thesis [11]. Several of the above articles investigate fragments of first-order theories by way of modal logics for affine, projective, and metric geometries. Our results contribute to the understanding of spatially motivated first-order languages, and hence they can be useful in the search for decidable (modal) spatial logics.

In addition to studying issues of decidability, we briefly compare the expressivities of universal monadic second-order logic $\forall \mathrm{MSO}$ and weak universal monadic second-order logic $\forall$ WMSO. It is straightforward to observe that in general, the expressivities of $\forall \mathrm{MSO}$ and $\forall \mathrm{WMSO}$ are incomparable in a rather strong sense: $\forall \mathrm{MSO} \not \leq \mathrm{WMSO}$ and $\forall \mathrm{WMSO} \not \leq \mathrm{MSO}$. Here MSO and WMSO denote monadic second-order logic and weak monadic second-order logic, respectively. The result $\forall \mathrm{WMSO} \not \leq \mathrm{MSO}$ follows from known results (see [10] for example), and the result $\forall \mathrm{MSO} \not \leq \mathrm{WMSO}$ is more or less trivial to prove. While $\forall \mathrm{MSO}$ and $\forall \mathrm{WMSO}$ are incomparable in general, the situation changes when we consider expansions $\left(\mathbb{R}^{n}, \beta,\left(R_{i}\right)_{i \in I}\right)$ of the structure $\left(\mathbb{R}^{n}, \beta\right)$, i.e., structures embedded in the geometric structure $\left(\mathbb{R}^{n}, \beta\right)$. Here $\left(R_{i}\right)_{i \in I}$ is an arbitrary vocabulary and $I$ an arbitrary related index set. We show that over such structures, sentences of $\forall \mathrm{WMSO}$ translate into equivalent sentences of $\forall \mathrm{MSO}$. The result follows immediately from our proof that the expansion class $F\left(\left(\mathbb{R}^{n}, \beta\right)\right)$ is first-order definable with respect to the class $E\left(\left(\mathbb{R}^{n}, \beta\right)\right)$, see Section 3 . The proof is based on the Heine-Borel theorem.

The structure of the current article is as follows. In Section 2 we define the central notions needed in the later sections. In Section 3 we compare the expressivities of $\forall$ MSO and $\forall$ WMSO. In Section 4 we show undecidability of the first-order theory of the unary expansion class of any geometric structure $(T, \beta)$ such that $T$ extends linearly in $2 \mathrm{D}$. In addition, we show that for, $n \geq 2$, the first-order theory of the unary expansion class of $\left(\mathbb{R}^{n}, \beta\right)$ is not arithmetical. In Section 5 we modify the approach in Section 4 and show undecidability of the FO-theory of any class $F((T, \beta))$ such that $T$ extends linearly in 2D.

For further information about Tarski and the facts proved by his school regarding fragments of ordered affine geometry relevant to the current paper, see [27] and [29], and the papers [23] and [24]. For a comprehensive survey on the development of the axiomatics of geometries of order, see [22], which, among other things, summarizes the results of [23] and [24].

This article is an extended version of the conference paper [19].

\section{Preliminaries}

2.1. Interpretations. Let $\sigma$ be a purely relational vocabulary, i.e., a vocabulary that does not contain function symbols or constant symbols. Let $\tau$ be a vocabulary that does not contain function symbols. Let $\mathcal{B}$ be a nonempty class of $\sigma$-structures and $\mathcal{C}$ a nonempty class of $\tau$-structures. Assume that there exists a surjective map $F$ from $\mathcal{C}$ onto $\mathcal{B}$ and a 
first-order $\tau$-formula $\varphi_{\text {Dom }}(x)$ in one free variable, $x$, such that for each structure $\mathfrak{C} \in \mathcal{C}$, there is a bijection $f$ from the domain of $F(\mathfrak{C})$ to the set

$$
\left\{u \in \operatorname{Dom}(\mathfrak{C}) \mid \mathfrak{C} \models \varphi_{\operatorname{Dom}}(u)\right\} .
$$

Assume, furthermore, that for each relation symbol $R \in \sigma$, there is a first-order $\tau$-formula $\varphi_{R}\left(x_{1}, \ldots, x_{A r(R)}\right)$ such that we have

$$
R^{F(\mathfrak{C})}\left(u_{1}, \ldots, u_{A r(R)}\right) \Leftrightarrow \mathfrak{C} \mid=\varphi_{R}\left(f\left(u_{1}\right), \ldots, f\left(u_{A r(R)}\right)\right)
$$

for every tuple $\left(u_{1}, \ldots, u_{A r(R)}\right) \in(\operatorname{Dom}(F(\mathfrak{C})))^{\operatorname{Ar}(R)}$. Here $\operatorname{Ar}(R)$ is the arity of $R$. We then say that the class $\mathcal{B}$ is uniformly first-order interpretable in $\mathcal{C}$.

Assume that a class of $\sigma$-structures $\mathcal{B}$ is uniformly first-order interpretable in a class $\mathcal{C}$ of $\tau$-structures. Define a map $I$ from the set of first-order $\sigma$-formulae to the set of first-order $\tau$-formulae as follows.

(1) If $k \in \mathbb{N}_{\geq 1}$ and $R \in \sigma$ is a $k$-ary relation symbol, then

$$
I\left(R\left(x_{1}, \ldots, x_{k}\right)\right):=\varphi_{R}\left(x_{1}, \ldots, x_{k}\right),
$$

where $\varphi_{R}\left(x_{1}, \ldots, x_{k}\right)$ is a first-order formula for $R$ witnessing the fact that $\mathcal{B}$ is uniformly first-order interpretable in $\mathcal{C}$.

(2) $I(x=y):=x=y$.

(3) $I(\neg \varphi):=\neg I(\varphi)$.

(4) $I(\varphi \wedge \psi):=I(\varphi) \wedge I(\psi)$.

(5) $I(\exists x \psi(x)):=\exists x\left(\varphi_{\text {Dom }}(x) \wedge I(\psi(x))\right)$.

We call the map $I$ a uniform interpretation of $\mathcal{B}$ in $\mathcal{C}$. Also, if $\mathcal{A}$ is the class of reducts of structures $\mathfrak{B} \in \mathcal{B}$ to some vocabulary $\rho \subseteq \sigma$, the function $I$ is called a uniform interpretation of $\mathcal{A}$ in $\mathcal{C}$.

Lemma 2.1. Let $\rho$ be a purely relational vocabulary and $\tau$ a vocabulary not containing function symbols. Let $\mathcal{A}$ be a class of $\rho$-structure and $\mathcal{C}$ a class of $\tau$-structures. Let $I$ be a uniform interpretation of $\mathcal{A}$ in $\mathcal{C}$. Let $\varphi$ be a first-order formula of the vocabulary $\rho$. Then the following conditions are equivalent.

(1) There exists a structure $\mathfrak{A} \in \mathcal{A}$ such that $\mathfrak{A}=\varphi$.

(2) There exists a structure $\mathfrak{C} \in \mathcal{C}$ such that $\mathfrak{C}=I(\varphi)$.

Proof. Straightforward.

2.2. Logics and structures. Monadic second-order logic, MSO, extends first-order logic with quantification of relation symbols ranging over subsets of the domain of a model. In universal (existential) monadic second-order logic, $\forall \mathrm{MSO}(\exists \mathrm{MSO})$, the quantification of monadic relations is restricted to universal (existential) prenex quantification in the beginning of formulae. The logics $\forall \mathrm{MSO}$ and $\exists \mathrm{MSO}$ are also known as monadic $\Pi_{1}^{1}$ and monadic $\Sigma_{1}^{1}$, respectively. Weak monadic second-order logic, WMSO, is a semantic variant of monadic second-order logic in which the quantified relation symbols range over finite subsets of the domain of a model. The weak variants $\forall \mathrm{WMSO}$ and $\exists \mathrm{WMSO}$ of $\forall \mathrm{MSO}$ and $\exists \mathrm{MSO}$ are defined in the obvious way. For further information on MSO, see for example [12] and [20].

Monadic second-order logic can be characterized by a variant of the Ehrenfeucht-Fraïssé game. We will give a short description of the game here. A more detailed description can be 

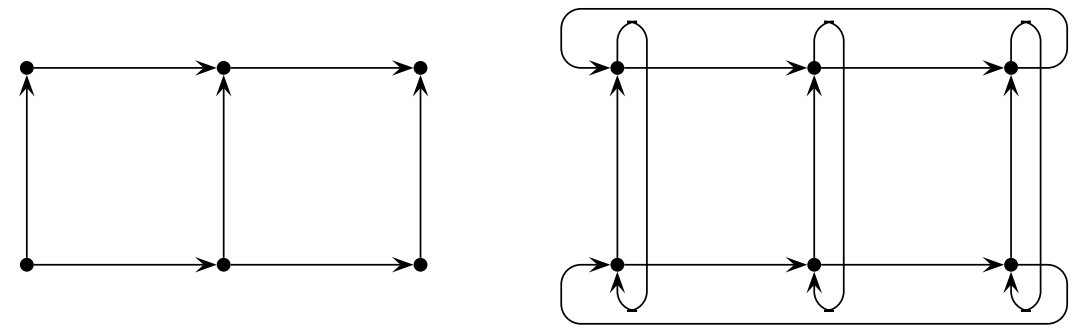

Figure 1: The figure shows a $3 \times 2$ grid and a $3 \times 2$ torus.

found in [20]. An MSO game is played by two players, the spoiler and the duplicator, on two structures $\mathfrak{A}$ and $\mathfrak{B}$ of the same purely relational vocabulary $\sigma$. A round starts by spoiler picking a structure, $\mathfrak{A}$ or $\mathfrak{B}$, and an element or a subset of that structure. The duplicator responds by choosing an object of the same type from the other structure. Let $\vec{a}$ and $\vec{b}$ be the elements and $\vec{A}$ and $\vec{B}$ the subsets chosen in a $k$-round game from the structures $\mathfrak{A}$ and $\mathfrak{B}$, respectively. Then the duplicator wins the game iff $(\vec{a}, \vec{b})$ defines a partial isomorphism from $(\mathfrak{A}, \vec{A})$ to $(\mathfrak{B}, \vec{B})$.

The $k$-round game characterizes $\mathrm{MSO}[k]$, the fragment of MSO up to the quantifier nesting depth $k$. More formally: If $\mathfrak{A}$ and $\mathfrak{B}$ are two structures of the same purely relational vocabulary, then the duplicator has a winning strategy in the $k$-round MSO game on $\mathfrak{A}$ and $\mathfrak{B}$ iff $\mathfrak{A}$ and $\mathfrak{B}$ agree on all sentences of $\operatorname{MSO}[k]$.

Let $\mathcal{L}$ be any fragment of second-order logic. The $\mathcal{L}$-theory of a structure $\mathfrak{M}$ of a vocabulary $\tau$ is the set of $\tau$-sentences $\varphi$ of $\mathcal{L}$ such that $\mathfrak{M} \models \varphi$.

Define two binary relations $H, V \subseteq \mathbb{N}^{2} \times \mathbb{N}^{2}$ as follows.

- $H=\{((i, j),(i+1, j)) \mid i, j \in \mathbb{N}\}$.

- $V=\{((i, j),(i, j+1)) \mid i, j \in \mathbb{N}\}$.

We let $\mathfrak{G}$ denote the structure $\left(\mathbb{N}^{2}, H, V\right)$, and call it the grid. The relations $H$ and $V$ are called the horizontal and vertical successor relations of $\mathfrak{G}$, respectively. A supergrid is a structure of the vocabulary $\{H, V\}$ that has $\mathfrak{G}$ as a substructure. We denote the class of supergrids by $\mathcal{G}$.

Let $(\mathfrak{G}, R)$ be the expansion of $\mathfrak{G}$, where $R=\left\{((0, i),(0, j)) \in \mathbb{N}^{2} \times \mathbb{N}^{2} \mid i<j\right\}$. We denote the structure $(\mathfrak{G}, R)$ by $\mathfrak{R}$, and call it the recurrence grid.

Let $m$ and $n$ be positive integers. Define two binary relations $H_{m, n}, V_{m, n} \subseteq(m \times n)^{2}$ as follows. (Note that we define $m=\{0, \ldots, m-1\}$, and analogously for $n$.)

- $H_{m, n}=H \uparrow(m \times n)^{2} \cup\{((m-1, i),(0, i)) \mid i<n\}$.

- $V_{m, n}=V \uparrow(m \times n)^{2} \cup\{((i, n-1),(i, 0)) \mid i<m\}$.

We call the structure $\left(m \times n, H_{m, n}, V_{m, n}\right)$ the $m \times n$ torus and denote it by $\mathfrak{T}_{m, n}$. A torus is essentially a finite grid whose east border wraps back to the west border and north border back to the south border.

2.3. Geometric affine betweenness structures. Let $\left(\mathbb{R}^{n}, d\right)$ be the $n$-dimensional Euclidean space with the canonical metric $d$. We always assume $n \geq 1$. We define the ternary Euclidean betweenness relation $\beta$ such that $\beta(s, t, u)$ iff

$$
d(s, u)=d(s, t)+d(t, u) .
$$


By $\beta^{*}$ we denote the strict betweenness relation, i.e., $\beta^{*}(s, t, u)$ iff $\beta(s, t, u)$ and $s \neq t \neq u$. We say that the points $s, t, u \in \mathbb{R}^{n}$ are collinear if the disjunction

$$
\beta(s, t, u) \vee \beta(s, u, t) \vee \beta(t, s, u)
$$

holds in $\left(\mathbb{R}^{n}, \beta\right)$. We define the first-order $\{\beta\}$-formula

$$
\operatorname{collinear}(x, y, z):=\beta(x, y, z) \vee \beta(x, z, y) \vee \beta(y, x, z) \text {. }
$$

Below we study geometric betweenness structures of the type $\left(T, \beta_{T}\right)$ where $T \subseteq \mathbb{R}^{n}$ and $\beta_{T}=\beta \uparrow T$. Here $\beta \uparrow T$ is the restriction of the betweenness predicate $\beta$ of $\mathbb{R}^{n}$ to the set $T$. To simplify notation, we usually refer to these structures by $(T, \beta)$.

Let $T \subseteq \mathbb{R}^{n}$ and let $\beta$ be the corresponding betweenness relation. We say that $L \subseteq T$ is a line in $T$ if the following conditions hold.

(1) There exist points $s, t \in L$ such that $s \neq t$.

(2) For all $s, t, u \in L$, the points $s, t, u$ are collinear.

(3) Let $s, t \in L$ be points such that $s \neq t$. For all $u \in T$, if $\beta(s, u, t)$ or $\beta(s, t, u)$, then $u \in L$.

Let $T \subseteq \mathbb{R}^{n}$ and let $L_{1}$ and $L_{2}$ be lines in $T$. We say that $L_{1}$ and $L_{2}$ intersect if $L_{1} \neq L_{2}$ and $L_{1} \cap L_{2} \neq \emptyset$. We say that the lines $L_{1}$ and $L_{2}$ intersect in $\mathbb{R}^{n}$ if $L_{1} \neq L_{2}$ and $L_{1}^{\prime} \cap L_{2}^{\prime} \neq \emptyset$, where $L_{1}^{\prime}, L_{2}^{\prime}$ are the lines in $\mathbb{R}^{n}$ such that $L_{1} \subseteq L_{1}^{\prime}$ and $L_{2} \subseteq L_{2}^{\prime}$.

A subset $S \subseteq \mathbb{R}^{n}$ is an $m$-dimensional flat of $\mathbb{R}^{n}$, where $0 \leq m \leq n$, if there exists a linearly independent set of $m$ vectors $v_{1}, \ldots, v_{m} \in \mathbb{R}^{n}$ and a vector $h \in \mathbb{R}^{n}$ such that $S$ is the $h$-translated span of the vectors $v_{1}, \ldots, v_{m}$, in other words

$$
S=\left\{u \in \mathbb{R}^{n} \mid u=h+r_{1} v_{1}+\cdots+r_{m} v_{m}, r_{1}, \ldots, r_{m} \in \mathbb{R}\right\} .
$$

None of the vectors $v_{i}$ is allowed to be the zero-vector. (This is relevant in the case where $m=1$.)

A nonempty set $U \subseteq \mathbb{R}^{n}$ is a linearly regular $m$-dimensional flat, where $0 \leq m \leq n$, if the following conditions hold.

(1) There exists an $m$-dimensional flat $S$ such that $U \subseteq S$.

(2) There does not exist any $(m-1)$-dimensional flat $S$ such that $U \subseteq S$.

(3) $U$ is linearly complete, i.e., if $L$ is a line in $U$ and $L^{\prime} \supseteq L$ the corresponding line in $\mathbb{R}^{n}$, and if $r \in L^{\prime}$ is a point in $L^{\prime}$ and $\epsilon \in \mathbb{R}_{+}$a positive real number, then there exists a point $s \in L$ such that $d(s, r)<\epsilon$. Here $d$ is the canonical metric of $\mathbb{R}^{n}$.

(4) $U$ is linearly closed, i.e., if $L_{1}$ and $L_{2}$ are lines in $U$ and $L_{1}$ and $L_{2}$ intersect in $\mathbb{R}^{n}$, then the lines $L_{1}$ and $L_{2}$ intersect. In other words, there exists a point $s \in U$ such that $s \in L_{1} \cap L_{2}$.

A set $T \subseteq \mathbb{R}^{n}$ extends linearly in $m D$, where $m \leq n$, if there exists a linearly regular $m$-dimensional flat $S$, a positive real number $\epsilon \in \mathbb{R}_{+}$and a point $x \in S \cap T$ such that $\{u \in S \mid d(x, u)<\epsilon\} \subseteq T$. It is easy show that for example $\mathbb{Q}^{2}$ extends linearly in $2 \mathrm{D}$.

Let $T \subseteq \mathbb{R}^{n}, n \in \mathbb{N}$ and let $\beta$ be the corresponding betweenness relation. The class of all expansions of $(T, \beta)$ to the vocabulary $\{\beta, P\}$, where $P$ is a unary relation symbol, is called the unary expansion class of $(T, \beta)$. By the unary expansion class of $(T, \beta)$ with a finite predicate, we mean the class of all expansions of $(T, \beta)$ to the vocabulary $\{\beta, P\}$, where the interpretation of $P$ is a finite set. 
2.4. Tilings. A function $t: 4 \longrightarrow \mathbb{N}$ is called a tile type. Define the set

$$
\text { TILESYMB }:=\left\{P_{t} \mid t \text { is a tile type }\right\}
$$

of unary relation symbols. The unary relation symbols in the set TILESYMB are called tile symbols. The numbers $t(i)$ of a tile symbol $P_{t}$ are the colours of $P_{t}$. The number $t(0)$ is the top colour, $t(1)$ the right colour, $t(2)$ the bottom colour, and $t(3)$ the left colour of $P_{t}$.

We then define a lexicographic linear ordering of tile types. Let $t$ and $s$ be tile types. We define $s<t$, if the tuple $(s(0), s(1), s(2), s(3))$ is situated below the tuple $(t(0), t(1), t(2), t(3))$ with respect to the canonical lexicographic ordering, i.e., $s<t$ if there exists some $i \in 4$ such that

(1) $s(i)<t(i)$, and

(2) $s(j)=t(j)$ for all $j$ such that $j<i$ and $j \in 4$.

If $t$ is a tile type, define $N(t)$ to be the number of tile types $s$ such that $s \leq t$. The function $N$ associates each tile type with a unique positive integer.

Let $T$ be a finite nonempty set of tile symbols. We say that a structure $\mathfrak{A}=(A, V, H)$, where $V, H \subseteq A^{2}$, is $T$-tilable, if there exists an expansion of $\mathfrak{A}$ to the vocabulary

$$
\{H, V\} \cup\left\{P_{t} \mid P_{t} \in T\right\}
$$

such that the following conditions hold.

(1) Each point of $A$ belongs to the extension of exactly one symbol $P_{t}$ in $T$.

(2) If $u H v$ for some points $u, v \in A$, then the right colour of the tile symbol $P_{t}$ s.t. $P_{t}(u)$ is the same as the left colour of the tile symbol $P_{t^{\prime}}$ such that $P_{t^{\prime}}(v)$.

(3) If $u V v$ for some points $u, v \in A$, then the top colour of the tile symbol $P_{t}$ s.t. $P_{t}(u)$ is the same as the bottom colour of the tile symbol $P_{t^{\prime}}$ such that $P_{t^{\prime}}(v)$.

Let $s$ be a tile type such that $P_{s} \in T$. We say that the grid $\mathfrak{G}$ is $s$-recurrently T-tilable, if there exists an expansion of $\mathfrak{G}$ to the vocabulary

$$
\{H, V\} \cup\left\{P_{t} \mid P_{t} \in T\right\}
$$

such that the above conditions (1) - (3) hold, and additionally, there exist infinitely many points $(0, i) \in \mathbb{N}^{2}$ such that $P_{s}((0, i))$. Intuitively, this means that the tile symbol $P_{s}$ occurs infinitely many times in the leftmost column of the grid $\mathfrak{G}$.

Let $\mathcal{F}$ be the set of finite, nonempty sets $T \subseteq$ TILESYMB, and let

$$
\mathcal{H}:=\left\{(t, T) \mid T \in \mathcal{F}, P_{t} \in T\right\} .
$$

Define the following languages

$$
\begin{aligned}
\mathcal{T} & :=\{T \in \mathcal{F} \mid \mathfrak{G} \text { is } T \text {-tilable }\}, \\
\mathcal{R} & :=\{(t, T) \in \mathcal{H} \mid \mathfrak{G} \text { is } t \text {-recurrently } T \text {-tilable }\}, \\
\mathcal{S} & :=\{T \in \mathcal{F} \mid \text { there is a torus } \mathfrak{D} \text { which is } T \text {-tilable }\} .
\end{aligned}
$$

The tiling problem is the membership problem of the set $\mathcal{T}$ with the input set $\mathcal{F}$. The recurrent tiling problem is the membership problem of the set $\mathcal{R}$ with the input set $\mathcal{H}$. The periodic tiling problem is the membership problem of $\mathcal{S}$ with the input set $\mathcal{F}$.

Theorem 2.2. 7] The tiling problem is $\Pi_{1}^{0}$-complete.

Theorem 2.3. [15] The recurrent tiling problem is $\Sigma_{1}^{1}$-complete.

Theorem 2.4. 13] The periodic tiling problem is $\Sigma_{1}^{0}$-complete. 
Lemma 2.5. There is a computable function associating each input $T$ to the (periodic) tiling problem with a first-order sentence $\varphi_{T}$ of the vocabulary $\tau:=\{H, V\} \cup T$ such that for all structures $\mathfrak{A}$ of the vocabulary $\{H, V\}$, the structure $\mathfrak{A}$ is T-tilable iff there exists an expansion $\mathfrak{A}^{*}$ of $\mathfrak{A}$ to the vocabulary $\tau$ such that $\mathfrak{A}^{*} \models \varphi_{T}$.

Proof. Straightforward.

Lemma 2.6. There is a computable function associating each input $(t, T)$ of the recurrent tiling problem with a first-order sentence $\varphi_{(t, T)}$ of the vocabulary $\tau:=\{H, V, R\} \cup T$ such that the grid $\mathfrak{G}$ is t-recurrently $T$-tilable iff there exists an expansion $\mathfrak{R}^{*}$ of the recurrence grid $\mathfrak{R}$ to the vocabulary $\tau$ such that $\mathfrak{R}^{*} \models \varphi_{(t, T)}$.

Proof. Straightforward.

It is easy to see that the grid $\mathfrak{G}$ is $T$-tilable iff there exists a supergrid $\mathfrak{G}^{\prime}$ that is $T$-tilable.

\section{Expressivity of UNIVERSAL MSO AND WEAK UNIVERSAL MSO OVER AFFINE REAL STRUCTURES $\left(\mathbb{R}^{n}, \beta\right)$}

In this section we investigate the expressive powers of $\forall \mathrm{WMSO}$ and $\forall \mathrm{MSO}$. While it is rather easy to conclude that the two logics are incomparable in a rather strong sense (see Proposition 3.1), when attention is limited to structures $\left(\mathbb{R}^{n}, \beta,\left(R_{i}\right)_{i \in I}\right)$ that expand the affine real structure $\left(\mathbb{R}^{n}, \beta\right)$, sentences of $\forall \mathrm{WMSO}$ translate into equivalent sentences of $\forall \mathrm{MSO}$.

Let $\mathcal{L}$ and $\mathcal{L}^{\prime}$ be fragments of second-order logic. We write $\mathcal{L} \leq \mathcal{L}^{\prime}$, if for every vocabulary $\sigma$, any class of $\sigma$-structures definable by a $\sigma$-sentence of $\mathcal{L}$ is also definable by a $\sigma$-sentence of $\mathcal{L}^{\prime}$. Let $\tau$ be a vocabulary such that $\beta \notin \tau$. The class of all expansions of $\left(\mathbb{R}^{n}, \beta\right)$ to the vocabulary $\{\beta\} \cup \tau$ is called the class of affine real $\tau$-structures. Such structures can be regarded as $\tau$-structures embedded in the geometric structure $\left(\mathbb{R}^{n}, \beta\right)$. We say that $\mathcal{L} \leq \mathcal{L}^{\prime}$ over $\left(\mathbb{R}^{n}, \beta\right)$, if for every vocabulary $\tau$ s.t. $\beta \notin \tau$, any subclass definable w.r.t. the class $\mathcal{C}$ of all affine real $\tau$-structures by a sentence of $\mathcal{L}$ is also definable w.r.t. $\mathcal{C}$ by a sentence of $\mathcal{L}^{\prime}$.

We sketch a canonical proof of the following very simple proposition. The result $\forall \mathrm{WMSO} \not \leq \mathrm{MSO}$ follows from already known results (see [10] for example), and the result $\forall \mathrm{MSO} \not \leq \mathrm{WMSO}$ is easy to prove.

Proposition 3.1. $\forall \mathrm{WMSO} \not \leq \mathrm{MSO}$ and $\forall \mathrm{MSO} \not \leq \mathrm{WMSO}$.

Proof Sketch. It is easy to observe that $\forall \mathrm{WMSO} \not \leq \mathrm{MSO}$ : consider the sentence $\forall X \exists y \neg X y$. This $\forall$ WMSO sentence is true in a model iff the domain of the model is infinite. A straightforward monadic second-order Ehrenfeucht-Fraïssé game argument can be used to establish that infinity is not expressible by any MSO sentence.

To show that $\forall \mathrm{MSO} \not \leq \mathrm{WMSO}$, consider the structures $(\mathbb{R},<)$ and $(\mathbb{Q},<)$. The structures can be separated by a sentence of $\forall \mathrm{MSO}$ stating that every subset bounded from above has a least upper bound. To see that the two structures cannot be separated by any sentence of WMSO, consider the variant of the MSO Ehrenfeucht-Fraïssé game where the players choose finite sets in addition to domain elements. It is easy to establish that this game characterizes the expressivity of WMSO. To see that the duplicator has a winning strategy in a game of any finite length played on the structures $(\mathbb{R},<)$ and $(\mathbb{Q},<)$, we devise an extension of the folklore winning strategy in the corresponding first-order game. Firstly, the duplicator can obviously always pick an element whose betweenness configuration corresponds exactly to 
that of the element picked by the spoiler. Furthermore, even if the spoiler picks a finite set, it is easy to see that the duplicator can pick his set such that each of its elements respect the betweenness configuration of the set picked by the spoiler.

We then show that $\forall \mathrm{WMSO} \leq \forall \mathrm{MSO}$ and WMSO $\leq \mathrm{MSO}$ over $\left(\mathbb{R}^{n}, \beta\right)$ for any $n \geq 1$.

Theorem 3.2 (Heine-Borel). A set $S \subseteq \mathbb{R}^{n}$ is closed and bounded iff every open cover of $S$ has a finite subcover.

Theorem 3.3. Let $\mathcal{C}$ be the class of expansions $\left(\mathbb{R}^{n}, \beta, P\right)$ of $\left(\mathbb{R}^{n}, \beta\right)$ with a unary predicate $P$, and let $\mathcal{F} \subseteq \mathcal{C}$ be the subclass of $\mathcal{C}$ where $P$ is finite. The class $\mathcal{F}$ is first-order definable with respect to $\mathcal{C}$.

Proof. We shall first establish that a set $T \subseteq \mathbb{R}^{n}$ is finite iff it is closed, bounded and consists of isolated points of $T$. Recall that an isolated point $u$ of a set $U \subseteq \mathbb{R}^{n}$ is a point such that there exists some open ball $B$ such that $B \cap U=\{u\}$.

Assume $T \subseteq \mathbb{R}^{n}$ is finite. Since $T$ is finite, we can find a minimum distance between points in the set $T$. Therefore it is clear that each point $t$ in $T$ belongs to some open ball $B$ such that $B \cap T=\{t\}$, and hence $T$ consists of isolated points. Similarly, since $T$ is finite, each point $b$ in the complement of $T$ has some minimum distance to the points of $T$, and therefore $b$ belongs to some open ball $B \subseteq \mathbb{R}^{n} \backslash T$. Hence the set $T$ is the complement of the union of open balls $B$ such that $B \subseteq \mathbb{R}^{n} \backslash T$, and therefore $T$ is closed. Finally, since $T$ is finite, we can find a maximum distance between the points in $T$, and therefore $T$ is bounded.

Assume then that $T \subseteq \mathbb{R}^{n}$ is closed, bounded and consists of isolated points of $T$. Since $T$ consists of isolated points, it has an open cover $\mathcal{C} \subseteq \operatorname{Pow}\left(\mathbb{R}^{n}\right)$ such that each set in $\mathcal{C}$ contains exactly one point $t \in T$. The set $\mathcal{C}$ is an open cover of $T$, and by the Heine-Borel theorem, there exists a finite subcover $\mathcal{D} \subseteq \mathcal{C}$ of the set $T$. Since $\mathcal{D}$ is finite and each set in $\mathcal{D}$ contains exactly one point of $T$, the set $T$ must also be finite.

We then conclude the proof by establishing that there exists a first-order formula $\varphi(P)$ stating that the unary predicate $P$ is closed, bounded and consists of isolated points. We will first define a formula parallel $(x, y, t, k)$ stating that the lines defined by $x, y$ and $t, k$ are parallel in $\left(\mathbb{R}^{n}, \beta\right)$. We define

$$
\begin{aligned}
& \text { parallel }(x, y, t, k):=x \neq y \wedge t \neq k \wedge((\operatorname{collinear}(x, y, t) \wedge \operatorname{collinear}(x, y, k)) \\
& \quad \vee(\neg \exists z(\operatorname{collinear}(x, y, z) \wedge \operatorname{collinear}(t, k, z)) \\
& \left.\left.\quad \wedge \exists z_{1} z_{2}\left(x \neq z_{1} \wedge \operatorname{collinear}\left(x, y, z_{1}\right) \wedge \operatorname{collinear}\left(x, t, z_{2}\right) \wedge \operatorname{collinear}\left(z_{1}, z_{2}, k\right)\right)\right)\right) .
\end{aligned}
$$

We will then define first-order $\{\beta\}$-formulae basis $_{k}\left(x_{0}, \ldots, x_{k}\right)$ and flat $_{k}\left(x_{0}, \ldots, x_{k}, z\right)$ using simultaneous recursion. The first formula states that the vectors corresponding to the pairs $\left(x_{0}, x_{i}\right), 1 \leq i \leq k$, form a basis of a $k$-dimensional flat. The second formula states that the point $z$ is in the span of the basis defined by the vectors $\left(x_{0}, x_{i}\right)$, the origin being $x_{0}$. First define basis $_{0}\left(x_{0}\right):=x_{0}=x_{0}$ and flat $x_{0}\left(x_{0}, z\right):=x_{0}=z$. Then define flat $_{k}$ and basis $k$ recursively in the following way.

$$
\begin{aligned}
\operatorname{basis}_{k}\left(x_{0}, \ldots, x_{k}\right) & :=\operatorname{basis}_{k-1}\left(x_{0}, \ldots, x_{k-1}\right) \wedge \neg \operatorname{flat}_{k-1}\left(x_{0}, \ldots, x_{k-1}, x_{k}\right), \\
\operatorname{flat}_{k}\left(x_{0}, \ldots, x_{k}, z\right) & :=\operatorname{basis}_{k}\left(x_{0}, \ldots, x_{k}\right) \\
\wedge & \exists y_{0} \ldots \exists y_{k}\left(y_{0}=x_{0} \wedge y_{k}=z \wedge \bigwedge_{i \leq k-1}\left(y_{i}=y_{i+1} \vee \operatorname{parallel}\left(x_{0}, x_{i+1}, y_{i}, y_{i+1}\right)\right)\right) .
\end{aligned}
$$




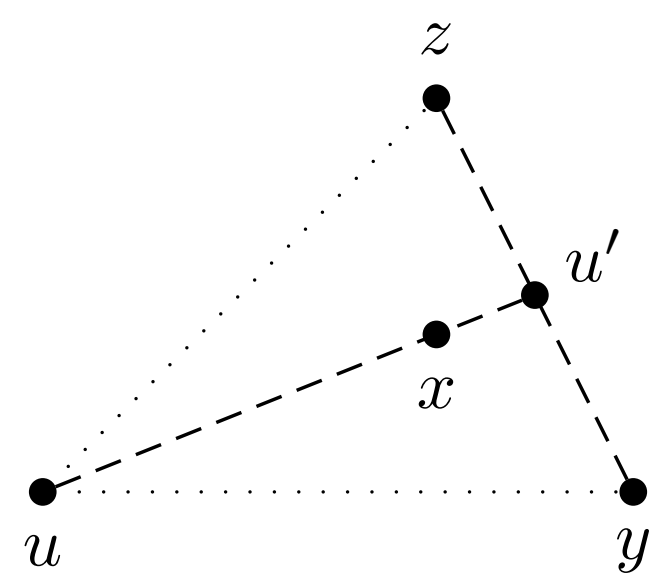

Figure 2: The figure shows how the formula opentriangle ${ }_{2}(y, z, u, x)$ is interpreted.

We then define a first-order $\{\beta, P\}$-formula $\operatorname{sepr}(x, P)$ asserting that $x$ belongs to an open ball $B$ such that each point in $B \backslash\{x\}$ belongs to the complement of $P$. The idea is to state that there exist $n+1$ points $x_{0}, \ldots, x_{n}$ that form an $n$-dimensional triangle around $x$, and every point contained in the triangle (with $x$ being a possible exception) belongs to the complement of $P$. Every open ball in $\mathbb{R}^{n}$ is contained in some $n$-dimensional triangle in $\mathbb{R}^{n}$ and vice versa. We will recursively define first-order formulae opentriangle ${ }_{k}\left(x_{0}, \ldots, x_{k}, z\right)$ stating that $z$ is properly inside a $k$-dimensional triangle defined by $x_{0}, \ldots, x_{k}$, see Figure 2 , First define opentriangle ${ }_{1}\left(x_{0}, x_{1}, z\right):=\beta^{*}\left(x_{0}, z, x_{1}\right)$, and then define

$$
\begin{aligned}
& \text { opentriangle }_{k}\left(x_{0}, \ldots, x_{k}, z\right):=\text { basis }_{k}\left(x_{0}, \ldots, x_{k}\right) \\
& \quad \wedge \exists y\left(\text { opentriangle }_{k-1}\left(x_{0}, \ldots, x_{k-1}, y\right) \wedge \beta^{*}\left(y, z, x_{k}\right)\right) .
\end{aligned}
$$

We are now ready to define $\operatorname{sepr}(x, P)$. Let

$$
\begin{aligned}
\operatorname{sepr}(x, P):=\exists x_{0} \ldots \exists x_{n}\left(\text { opentriangle }_{n}\left(x_{0}, \ldots, x_{n}, x\right)\right. & \\
& \left.\wedge \forall y\left(\left(\text { opentriangle }_{n}\left(x_{0}, \ldots, x_{n}, y\right) \wedge y \neq x\right) \rightarrow \neg P y\right)\right) .
\end{aligned}
$$

Now, the sentence $\varphi_{1}:=\forall x(\neg P x \rightarrow \operatorname{sepr}(x, P))$ states that each point in the complement of $P$ is contained in an open ball $B \subseteq \mathbb{R}^{n} \backslash P$. The sentence therefore states that the complement of $P$ is a union of open balls. Since the set of unions of open balls is exactly the same as the set of open sets, the sentence states that $P$ is closed.

The sentence $\varphi_{2}:=\forall x(P x \rightarrow \operatorname{sepr}(x, P))$ clearly states that $P$ consists of isolated points.

Finally, in order to state that $P$ is bounded, we define a formula asserting that there exist points $x_{0}, \ldots, x_{n}$ that form an n-dimensional triangle around $P$.

$$
\varphi_{3}:=\exists x_{0} \ldots \exists x_{n}\left(\operatorname{basis}_{n}\left(x_{0}, \ldots, x_{n}\right) \wedge \forall y\left(P y \rightarrow \text { opentriangle }_{n}\left(x_{0}, \ldots, x_{n}, y\right)\right)\right)
$$

The conjunction $\varphi_{1} \wedge \varphi_{2} \wedge \varphi_{3}$ states that $P$ is finite. 
Corollary 3.4. Limit attention to expansions of $\left(\mathbb{R}^{n}, \beta\right)$. Sentences of $\forall$ WMSO translate into equivalent sentences of $\forall \mathrm{MSO}$, and sentences of WMSO into equivalent sentences of MSO.

\section{UNDECIDABle theORIES OF GEOMETRIC STRUCTURES With AN AFFine BETWEENNESS} RELATION

In this section we establish undecidability of the first-order theory of the unary expansion class

$$
\{(T, \beta, P) \mid P \subseteq T\}
$$

of any geometric structure $(T, \beta)$ that extends linearly in $2 \mathrm{D}$. We also show that the first-order theories of unary expansion classes of structures $\left(\mathbb{R}^{n}, \beta\right)$ with $n \geq 2$ are highly undecidable. More precisely, we show that the theories of classes based on structures extending linearly in $2 \mathrm{D}$ are $\Sigma_{1}^{0}$-hard, while the theories of classes based on structures $\left(\mathbb{R}^{n}, \beta\right)$ with $n \geq 2$ are $\Pi_{1}^{1}$-hard - and therefore not even arithmetical. We establish the results by a reduction from the (recurrent) tiling problem to the problem of deciding whether a $\{\beta, P\}$-sentence is satisfied in some expansion $(T, \beta, P)$ of $(T, \beta)$ (respectively, in some expansion $\left(\mathbb{R}^{n}, \beta, P\right)$ of $\left.\left(\mathbb{R}^{n}, \beta\right)\right)$. The argument is based on interpreting supergrids in corresponding $\{\beta\}$-structures.

4.1. Lines and sequences. Let $T \subseteq \mathbb{R}^{n}$. Let $L$ be a line in $T$. Any nonempty subset $Q$ of $L$ is called a sequence in $T$. Let $E \subseteq T$ and $s, t \in T$. If $s \neq t$ and if $u \in E$ for all points $u \in T$ such that $\beta^{*}(s, u, t)$, we say that the points $s$ and $t$ are linearly E-connected (in $(T, \beta))$. If there exists a point $v \in T \backslash E$ such that $\beta^{*}(s, v, t)$, we say that $s$ and $t$ are linearly disconnected with respect to $E$ (in $(T, \beta)$ ).

Definition 4.1. Let $Q$ be a sequence in $T \subseteq \mathbb{R}^{n}$. Suppose that for each $s, t \in Q$ such that $s \neq t$, there exists a point $u \in T \backslash\{s\}$ such that

(1) $\beta(s, u, t)$ and

(2) $\forall r \in T\left(\beta^{*}(s, r, u) \rightarrow r \notin Q\right)$, i.e., the points $s$ and $u$ are linearly $(T \backslash Q)$-connected.

Then we call $Q$ a discretely spaced sequence in $T$.

Definition 4.2. Let $Q$ be a discretely spaced sequence in $T \subseteq \mathbb{R}^{n}$. Assume that there exists a point $s \in Q$ such that for each point $u \in Q$, there exists a point $v \in Q \backslash\{u\}$ such that $\beta(s, u, v)$. Then we call the sequence $Q$ a discretely infinite sequence in $T$. The point $s$ is called a base point of $Q$.

Definition 4.3. Let $Q$ be a sequence in $T \subseteq \mathbb{R}^{n}$. Let $s \in Q$ be a point such that there do not exist points $u, v \in Q \backslash\{s\}$ such that $\beta(u, s, v)$. Then we call $Q$ a sequence in $T$ with $a$ zero. The point $s$ is a zero-point of $Q$. Notice that $Q$ may have up to two zero-points.

It is easy to see that a discretely infinite sequence has at most one zero-point.

Definition 4.4. Let $Q$ be a discretely infinite sequence in $T \subseteq \mathbb{R}^{n}$ with a zero. Assume that for each $r \in T$ such that there exist points $s, u \in Q \backslash\{r\}$ with $\beta(s, r, u)$, there also exist points $s^{\prime}, u^{\prime} \in Q \backslash\{r\}$ such that

(1) $\beta\left(s^{\prime}, r, u^{\prime}\right)$ and

(2) $\forall v \in T \backslash\{r\}\left(\beta^{*}\left(s^{\prime}, v, u^{\prime}\right) \rightarrow v \notin Q\right)$.

Then we call $Q$ an $\omega$-like sequence in $T$ (cf. Lemma 4.7). 
Lemma 4.5. Let $P$ be a unary relation symbol. There is a first-order sentence $\varphi_{\omega}(P)$ of the vocabulary $\{\beta, P\}$ such that for every $T \subseteq \mathbb{R}^{n}$ and for every expansion $(T, \beta, P)$ of $(T, \beta)$, we have $(T, \beta, P) \models \varphi_{\omega}(P)$ if and only if the interpretation of $P$ is an $\omega$-like sequence in $T$.

Proof. Define

$$
\text { sequence }(P):=\exists x P x \wedge \forall x \forall y \forall z(P x \wedge P y \wedge P z \rightarrow \operatorname{collinear}(x, y, z)) .
$$

The formula sequence $(P)$ states that $P$ is a sequence. By inspection of Definition 4.1 , it is easy to see that there is a first-order formula $\psi$ such that the conjunction sequence $(P) \wedge \psi$ states that $P$ is a discretely spaced sequence. Continuing this trend, it is straightforward to observe that Definitions 4.2 , 4.3 and 4.4 specify first-order properties, and therefore there exists a first-order formula $\varphi_{\omega}(P)$ stating that $P$ is an $\omega$-like sequence.

Definition 4.6. Let $P$ be a sequence in $T \subseteq \mathbb{R}^{n}$ and $s, t \in P$. The points $s, t$ are called adjacent with respect to $P$, if the points are linearly $(T \backslash P)$-connected. Let $E \subseteq P \times P$ be the set of pairs $(u, v)$ such that

(1) $u$ and $v$ are adjacent with respect to $P$, and

(2) $\beta(z, u, v)$ for some zero-point $z$ of $P$.

We call $E$ the successor relation of $P$.

We let succ denote the successor relation of $\mathbb{N}$, i.e., succ $:=\{(i, j) \in \mathbb{N} \times \mathbb{N} \mid i+1=j\}$.

Lemma 4.7. Let $P$ be an $\omega$-like sequence in $T \subseteq \mathbb{R}^{n}$ and $E$ the successor relation of $P$. There is an embedding from $(\mathbb{N}$, succ $)$ into $(P, E)$ such that $0 \in \mathbb{N}$ maps to the zero-point of $P$. If $T=\mathbb{R}^{n}$, then $(\mathbb{N}$, succ $)$ is isomorphic to $(P, E)$.

Proof. We denote by $i_{0}$ the unique zero-point of $P$. Since $P$ is a discretely infinite sequence, it has a base point. Clearly $i_{0}$ has to be the only base point of $P$. It is straightforward to establish that since $P$ is an $\omega$-like sequence with the base point $i_{0}$, there exists a sequence $\left(a_{i}\right)_{i \in \mathbb{N}}$ of points $a_{i} \in P$ such that $i_{0}=a_{0}$ and $a_{i+1}$ is the unique $E$-successor of $a_{i}$ for all $i \in \mathbb{N}$. Define the function $h: \mathbb{N} \rightarrow P$ such that $h(i)=a_{i}$ for all $i \in \mathbb{N}$. It is easy to see that $h$ is an embedding of $(\mathbb{N}$, succ $)$ into $(P, E)$.

Assume then that $T=\mathbb{R}^{n}$. We shall show that the function $h: \mathbb{N} \longrightarrow P$ is a surjection. Let $d$ denote the canonical metric of $\mathbb{R}$, and let $d_{R}$ be the restriction of the canonical metric of $\mathbb{R}^{n}$ to the line $R$ in $\mathbb{R}^{n}$ such that $P \subseteq R$. Let $g: \mathbb{R} \longrightarrow R$ be the isometry from $(\mathbb{R}, d)$ to $\left(R, d_{R}\right)$ such that $g(0)=i_{0}=h(0)$ and such that for all $r \in \operatorname{ran}(h)$, we have $\beta\left(i_{0}, g(1), r\right)$ or $\beta\left(i_{0}, r, g(1)\right)$. Let $\left(R, \leq^{R}\right)$ be the structure, where

$$
\leq^{R}=\left\{(u, v) \in R \times R \mid g^{-1}(u) \leq^{\mathbb{R}} g^{-1}(v)\right\} .
$$

If $\operatorname{ran}(h)$ is not bounded from above w.r.t. $\leq^{R}$, then $h$ must be a surjection. Therefore assume that $\operatorname{ran}(h)$ is bounded above. By the Dedekind completeness of the reals, there exists a least upper bound $s \in R$ of $\operatorname{ran}(h)$ w.r.t. $\leq^{R}$. Notice that since $h$ is an embedding of $(\mathbb{N}$, succ $)$ into $(P, E)$, we have $s \notin \operatorname{ran}(h)$. Due to the definition of $E$, it is sufficient to show that $\left\{t \in P \mid s \leq^{R} t\right\}=\emptyset$ in order to conclude that $h$ maps onto $P$.

Assume that the least upper bound $s$ belongs to the set $P$. Since $P$ is a discretely spaced sequence, there is a point $u \in \mathbb{R}^{n} \backslash\{s\}$ such that $\beta\left(s, u, i_{0}\right)$ and

$$
\forall r \in \mathbb{R}^{n}\left(\beta^{*}(s, r, u) \rightarrow r \notin P\right) .
$$

Now $u<^{R} s$ and the points $u$ and $s$ are linearly $\left(\mathbb{R}^{n} \backslash P\right)$-connected, implying that $s$ cannot be the least upper bound of $\operatorname{ran}(h)$. This is a contradiction. Therefore $s \notin P$. 


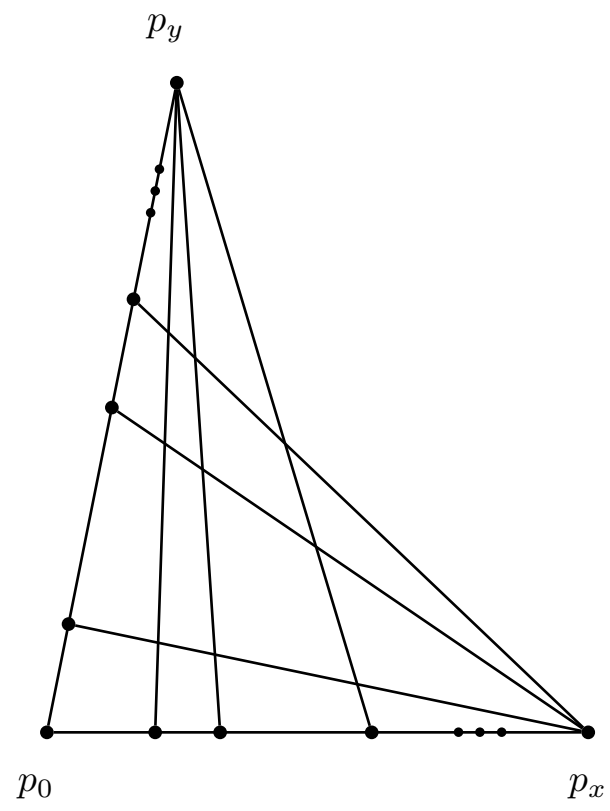

Figure 3: The figure illustrates how a grid is interpreted in a Cartesian frame. The intersection points of the solid lines correspond to domain points of the grid. See also figure 4 .

Assume, ad absurdum, that there exists a point $t \in P$ such that $\beta\left(i_{0}, s, t\right)$. Now, since $P$ is an $\omega$-like sequence, there exists points $u^{\prime}, v^{\prime} \in P \backslash\{s\}$ such that $\beta\left(u^{\prime}, s, v^{\prime}\right)$ and

$$
\forall r \in \mathbb{R}^{n}\left(\beta^{*}\left(u^{\prime}, r, v^{\prime}\right) \rightarrow r \notin P\right) .
$$

We have $\beta\left(s, u^{\prime}, i_{0}\right)$ or $\beta\left(s, v^{\prime}, i_{0}\right)$. Assume, by symmetry, that $\beta\left(s, u^{\prime}, i_{0}\right)$. Now $u^{\prime}<^{R} s$, and the points $u^{\prime}$ and $s$ are linearly $\left(\mathbb{R}^{n} \backslash P\right)$-connected. Hence, since $s \notin \operatorname{ran}(h)$, we conclude that $s$ is not the least upper bound of $\operatorname{ran}(h)$. This is a contradiction.

4.2. Geometric structures with an undecidable unary expansion class. Let $Q$ be an $\omega$-like sequence in $T \subseteq \mathbb{R}^{n}$ and let $q_{0}$ be the unique zero-point of $Q$. Assume there exists a point $q_{e} \in T \backslash Q$ such that $\beta\left(q_{0}, q, q_{e}\right)$ holds for all $q \in Q$. We call $Q \cup\left\{q_{e}\right\}$ an $\omega$-like sequence with an endpoint in $T$. The point $q_{e}$ is the endpoint of $Q \cup\left\{q_{e}\right\}$. Notice that the endpoint $q_{e}$ is the only point $x$ in $Q \cup\left\{q_{e}\right\}$ such that the following conditions hold.

(1) There do not exist points $s, t \in Q \cup\left\{q_{e}\right\}$ such that $\beta^{*}(s, x, t)$.

(2) $\forall y z \in Q \cup\left\{q_{e}\right\}\left(\beta^{*}(x, y, z) \rightarrow \exists v \in Q \cup\left\{q_{e}\right\} \beta^{*}(x, v, y)\right)$.

Definition 4.8. Let $P \subseteq T \subseteq \mathbb{R}^{n}$, and let $p_{0}, p_{x}, p_{y} \in P$. We call the structure

$$
\mathfrak{C}=\left(T, \beta, P, p_{0}, p_{x}, p_{y}\right)
$$

a Cartesian frame with domain $T$, if the following conditions hold.

(1) The points $p_{0}, p_{x}$ and $p_{y}$ are not collinear, i.e., collinear $\left(p_{0}, p_{x}, p_{y}\right)$ does not hold in the structure $\mathfrak{C}$. 


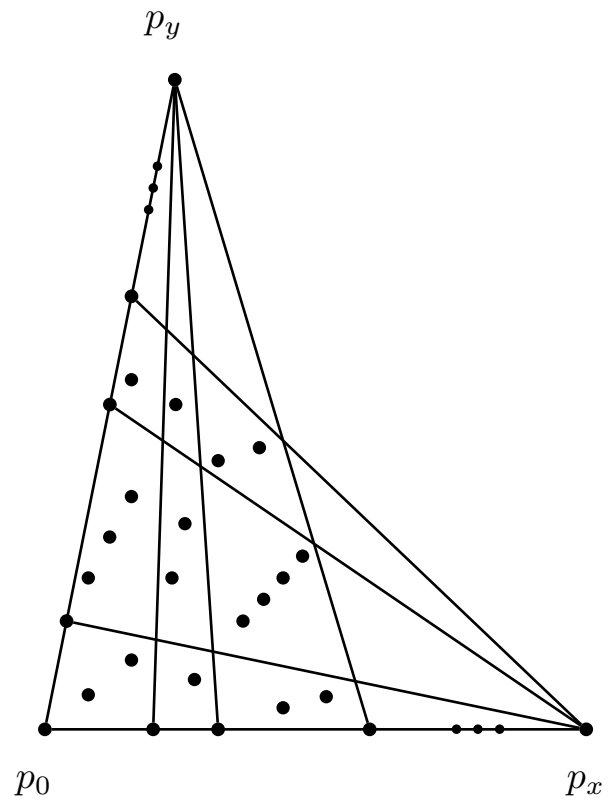

Figure 4: The figure shows how the tile symbols of a labelled grid are interpreted in a Cartesian frame. Each tile symbol $P_{t}$ is associated with the natural number $N(t)$ (see the Preliminaries section). The number of dots $n$ on the southwest to northeast diagonal of the rectangle to the northeast of an intersection point $x$ corresponds to the tile symbol associated with $x$. The point $x$ is associated with $P_{t}$ iff $n=N(t)$.

(2) The set

$$
P_{x}=\left\{u \in P \mid \operatorname{collinear}\left(p_{0}, u, p_{x}\right) \text { holds in } \mathfrak{C}\right\}
$$

is an $\omega$-like sequence with an endpoint in $T$. The point $p_{x}$ is the endpoint of $P_{x}$.

(3) The set

$$
P_{y}=\left\{u \in P \mid \operatorname{collinear}\left(p_{0}, u, p_{y}\right) \text { holds in } \mathfrak{C}\right\}
$$

is an $\omega$-like sequence with an endpoint in $T$. The point $p_{y}$ is the endpoint of $P_{y}$.

(4) The point $p_{0}$ is the zero-point of both $P_{x} \backslash\left\{p_{x}\right\}$ and $P_{y} \backslash\left\{p_{y}\right\}$.

(5) For each point $p \in P_{x} \backslash\left\{p_{x}\right\}$ and $q \in P_{y} \backslash\left\{p_{y}\right\}$, the unique lines $L_{p}$ and $L_{q}$ in $T$ such that $p, p_{y} \in L_{p}$ and $q, p_{x} \in L_{q}$, intersect. In other words, there exists a point $u \in T$ that lies on both lines $L_{p}$ and $L_{q}$.

Definition 4.9. Let $\mathfrak{C}=\left(T, \beta, P, p_{0}, p_{x}, p_{y}\right)$ be a Cartesian frame. Let $p \in P_{x} \backslash\left\{p_{x}\right\}$ and $q \in P_{y} \backslash\left\{p_{y}\right\}$ be points and $L_{p}$ and $L_{q}$ the lines in $T$ such that $p, p_{y} \in L_{p}$ and $q, p_{x} \in L_{q}$. The point $u \in T$ that lies on both lines $L_{p}$ and $L_{q}$ is called - rather suggestively - the intersection point of $\mathfrak{C}$ corresponding to the pair $(p, q)$. A point $u \in T$ is called an intersection point of the Cartesian frame $\mathfrak{C}$, if it is an intersection point of $\mathfrak{C}$ corresponding some pair $(p, q)$, where $p \in P_{x} \backslash\left\{p_{x}\right\}$ and $q \in P_{y} \backslash\left\{p_{y}\right\}$.

Definition 4.10. Let $\mathfrak{C}=\left(T, \beta, P, p_{0}, p_{x}, p_{y}\right)$ be a Cartesian frame. Recall Definition 4.6. Let $E_{x}$ be the successor relation of the $\omega$-like sequence $P_{x} \backslash\left\{p_{x}\right\}$ and $E_{y}$ the successor relation of $P_{y} \backslash\left\{p_{y}\right\}$. Let $p, p^{\prime}, q, q^{\prime}$ be points such that $\left(p, p^{\prime}\right) \in E_{x}$ and $\left(q, q^{\prime}\right) \in E_{y}$. Let 
$u$ be the intersection point of $\mathfrak{C}$ corresponding to $(p, q)$ and $v$ the intersection point of $\mathfrak{C}$ corresponding to $\left(p^{\prime}, q^{\prime}\right)$. We say that $v$ is the diagonal successor of $u$ in $\mathfrak{C}$.

Definition 4.11. Recall the function $N$ that associates each tile type $t$ with the unique positive integer $N(t)$ (see the Preliminaries section). Let $\mathfrak{C}=\left(T, \beta, P, p_{0}, p_{x}, p_{y}\right)$ be a Cartesian frame and let $S \neq \emptyset$ be a finite set of tile symbols. We call $\mathfrak{C}$ an $S$-labelled Cartesian frame if the number of points in $P$ strictly between any intersection point $u$ of $\mathfrak{C}$ and its diagonal successor $v$ is in the set $\left\{N(t) \mid P_{t} \in S\right\}$. If $T \subseteq \mathbb{R}^{n}$ and $S \neq \emptyset$ is a finite set of tile symbols, we let $\mathcal{C}(T, S)$ denote the class of exactly all $S$-labelled Cartesian frames with domain $T$.

Lemma 4.12. Let $T \subseteq \mathbb{R}^{n}, n \geq 2$, and let $\mathcal{C}$ be the class of all expansions $\left(T, \beta, P, p_{0}, p_{x}, p_{y}\right)$ of $(T, \beta)$ by a unary relation $P$ and constants $p_{0}, p_{x}, p_{y}$. There is a computable function associating each input $S$ to the tiling problem with a first-order $\left\{\beta, P, p_{0}, p_{x}, p_{y}\right\}$-sentence $\varphi_{C f}^{S}$ that defines the class of $S$-labelled Cartesian frames with the domain $T$ with respect to the class $\mathcal{C}$.

Proof. Straightforward by virtue of Lemma 4.5 .

Recall that we let $\mathfrak{G}$ denote the grid. Let $S \neq \emptyset$ be a finite set of tile symbols. We let $\mathfrak{G}_{S}$ denote the class of structures $\mathfrak{A}$ that satisfy the following conditions.

(1) The structure $\mathfrak{A}$ is an expansion of the grid $\mathfrak{G}$ to the vocabulary $\{V, H\} \cup S$.

(2) Each point in the domain of $\mathfrak{A}$ is in the extension of exactly one predicate symbol $P_{t} \in S$. Structures in the class $\mathfrak{G}_{S}$ are called $S$-labelled grids. Similarly, we let $\mathcal{G}_{S}$ denote the class of structures $\mathfrak{A}$ that satisfy the following conditions.

(1) The structure $\mathfrak{A}$ is an expansion of some supergrid to the vocabulary $\{V, H\} \cup S$.

(2) Each point in the domain of $\mathfrak{A}$ is in the extension of exactly one predicate symbol $P_{t} \in S$. Structures in the class $\mathcal{G}_{S}$ are called $S$-labelled supergrids. The class of $S$-labelled recurrence grids is defined in the obvious way.

Lemma 4.13. Let $T \subseteq \mathbb{R}^{n}$ be a set that extends linearly in $2 \mathrm{D}$. There is a computable function $I$ such that for each finite set of tile symbols $S \neq \emptyset$, there exists some class $\mathcal{G}(T, S) \supseteq \mathfrak{G}_{S}$ of $S$-labelled supergrids such that the function $I$ is a uniform interpretation of $\mathcal{G}(T, S)$ in $\mathcal{C}(T, S)$.

Proof. Let $S \neq \emptyset$ be a finite set of tile symbols. Let $\mathfrak{C}=\left(T, \beta, P, p_{0}, p_{x}, p_{y}\right)$ be an $S$-labelled Cartesian frame. We shall show how an $S$-labelled supergrid $\mathfrak{G}_{\mathfrak{C}}$ is interpreted in $\mathfrak{C}$. Figures 3 and 4 illustrate the related constructions.

The domain of the interpretation of $\mathfrak{G}_{\mathfrak{C}}$ in $\mathfrak{C}$ will be the set of intersection points of two sets of lines defined as follows. The first set of lines is formed by connecting the point $p_{y}$ to each of the points $u$ in the set

$$
\left\{x \in P \mid \beta\left(p_{0}, x, p_{x}\right)\right\} \backslash\left\{p_{x}\right\},
$$

i.e., each line in the set corresponds to a pair $\left(u, p_{y}\right)$ for some $u \neq p_{x}$ such that $\beta\left(p_{0}, u, p_{x}\right)$. Similarly, the second set of lines is formed by connecting the point $p_{x}$ to each of the points in the set

$$
\left\{x \in P \mid \beta\left(p_{0}, x, p_{y}\right)\right\} \backslash\left\{p_{y}\right\} .
$$


We then define the formulae

$$
\begin{aligned}
\varphi_{\text {Dom }}(u):= & \exists x y\left(P(x) \wedge P(y) \wedge \beta^{*}\left(p_{0}, x, p_{x}\right) \wedge \beta^{*}\left(p_{0}, y, p_{y}\right) \wedge \beta^{*}\left(x, u, p_{y}\right) \wedge \beta^{*}\left(y, u, p_{x}\right)\right) \\
& \vee\left(u \neq p_{x} \wedge u \neq p_{y} \wedge P(u) \wedge\left(\beta\left(p_{0}, u, p_{x}\right) \vee \beta\left(p_{0}, u, p_{y}\right)\right)\right), \\
\varphi_{H}(u, v):= & \exists x\left(\beta\left(p_{0}, x, p_{y}\right) \wedge \beta(x, u, v) \wedge \beta^{*}\left(u, v, p_{x}\right)\right) \wedge \forall r\left(\beta^{*}(u, r, v) \rightarrow \neg \varphi_{D o m}(r)\right), \\
\varphi_{V}(u, v):= & \exists x\left(\beta\left(p_{0}, x, p_{x}\right) \wedge \beta(x, u, v) \wedge \beta^{*}\left(u, v, p_{y}\right)\right) \wedge \forall r\left(\beta^{*}(u, r, v) \rightarrow \neg \varphi_{D o m}(r)\right) .
\end{aligned}
$$

Next we define the following auxiliary formula:

$$
\operatorname{diagonal}(u, v):=\exists x\left(\varphi_{\text {Dom }}(x) \wedge \varphi_{H}(u, x) \wedge \varphi_{V}(x, v)\right) .
$$

Recall the function $N$ that associates each tile type $t$ with the unique positive integer $N(t)$. Let $\exists^{=N(t)} x$ denote the quantifier stating that there exist exactly $N(t) x$ :s. Now, for each tile symbol $P_{t}$, we define

$$
\varphi_{P_{t}}(u):=\exists z \exists^{=N(t)} x\left(\varphi_{\text {Dom }}(z) \wedge \operatorname{diagonal}(u, z) \wedge P(x) \wedge \beta^{*}(u, x, z)\right) .
$$

The formulae $\varphi_{D o m}, \varphi_{H}, \varphi_{V}$ and $\varphi_{P(t)}$ define the uniform interpretation $I$.

Call $D_{\mathfrak{C}}:=\left\{u \in T \mid \mathfrak{C} \models \varphi_{\text {Dom }}(u)\right\}$, and define the structure

$$
\mathfrak{D}_{\mathfrak{C}}=\left(D_{\mathfrak{C}}, H^{\mathfrak{D}_{\mathfrak{C}}}, V^{\mathfrak{D}_{\mathfrak{C}}},\left(P_{t}^{\mathfrak{D}_{\mathfrak{C}}}\right)_{P_{t} \in S}\right),
$$

where

$$
\begin{aligned}
H^{\mathfrak{D}_{\mathfrak{C}}} & :=\left\{(u, v) \in D_{\mathfrak{C}} \times D_{\mathfrak{C}} \mid \mathfrak{C}=\varphi_{H}(u, v)\right\}, \\
V^{\mathfrak{D}_{\mathfrak{C}}} & :=\left\{(u, v) \in D_{\mathfrak{C}} \times D_{\mathfrak{C}} \mid \mathfrak{C}=\varphi_{V}(u, v)\right\}, \\
P_{t}^{\mathfrak{D}_{\mathfrak{C}}} & :=\left\{u \in D_{\mathfrak{C}} \mid \mathfrak{C}=\varphi_{P_{t}}(u)\right\} \text { for each } P_{t} \in S .
\end{aligned}
$$

By Lemma 4.7, it is easy to see that there is and $S$-labelled grid $\left(G, H, V,\left(P_{t}\right)_{P_{t} \in S}\right)$ such that there exists an injection $f$ from the domain of the grid to $D_{\mathfrak{C}}$ such that the following three conditions hold for all $u, v \in G$ and $P_{t} \in S$ :

(1) $(u, v) \in H \Leftrightarrow \varphi_{H}(f(u), f(v))$,

(2) $(u, v) \in V \Leftrightarrow \varphi_{V}(f(u), f(v))$,

(3) $u \in P_{t} \Leftrightarrow \varphi_{P_{t}}(f(u))$.

Hence there is an $S$-labelled supergrid $\mathfrak{G}_{\mathfrak{C}}=\left(G_{\mathfrak{C}}, H^{\mathfrak{G}_{\mathfrak{C}}}, V^{\mathfrak{G}_{\mathfrak{C}}},\left(P_{t}^{\mathfrak{G}_{\mathfrak{C}}}\right)_{P_{t} \in S}\right)$ such that there exists a bijection $f$ from $G_{\mathfrak{C}}$ to $D_{\mathfrak{C}}$ such that the following conditions hold for all $u, v \in G_{\mathfrak{C}}$ and $P_{t} \in S$ :

(1) $(u, v) \in H^{\mathfrak{G}_{\mathfrak{C}}} \Leftrightarrow \varphi_{H}(f(u), f(v))$,

(2) $(u, v) \in V^{\mathfrak{G}_{\mathfrak{C}}} \Leftrightarrow \varphi_{V}(f(u), f(v))$,

(3) $u \in P_{t}^{\mathfrak{G}_{\mathfrak{C}}} \Leftrightarrow \varphi_{P_{t}}(f(u))$.

Let

$$
\mathcal{G}(T, S):=\left\{\mathfrak{G}_{\mathfrak{C}} \in \mathcal{G}_{S} \mid \mathfrak{C} \in \mathcal{C}(T, S)\right\} .
$$

Since $T$ extends linearly in $2 \mathrm{D}$, we have $\mathfrak{G}_{S} \subseteq \mathcal{G}(T, S)$. The function $I$ is a uniform interpretation of $\mathcal{G}(T, S)$ in $\mathcal{C}(T, S)$.

Lemma 4.14. Let $n \geq 2$ be an integer. There is a computable function $K$ such that for each nonempty set $S$ of tile symbols, the function $K$ is a uniform interpretation of the class of S-labelled recurrence grids in the class of $S$-labelled Cartesian frames with domain $\mathbb{R}^{n}$.

Proof. Straightforward by Lemma 4.7 and the proof of Lemma 4.13. 
Theorem 4.15. Let $T \subseteq \mathbb{R}^{n}$ be a set and $\beta$ be the corresponding betweenness relation. Assume that $T$ extends linearly in 2D. The first-order theory of the unary expansion class of $(T, \beta)$ is $\Sigma_{1}^{0}$-hard.

Proof. Since $T$ extends linearly in $2 \mathrm{D}$, we have $n \geq 2$. Let $\sigma=\{H, V\}$ be the vocabulary of supergrids, and let $\tau=\left\{\beta, P, p_{0}, p_{x}, p_{y}\right\}$ be the vocabulary of labelled Cartesian frames. By Lemma 4.12, there is a computable function that associates each input $S$ to the tiling problem with a first-order $\tau$-sentence that defines the class of $S$-labelled Cartesian frames with the domain $T$ with respect to the class of all expansions of $(T, \beta)$ to the vocabulary $\tau$. Let $\varphi_{C f}^{S}$ denote such a sentence. By Lemma 2.5. there is a computable function that associates each input $S$ to the tiling problem with a first-order $\sigma \cup S$-sentence $\varphi_{S}$ such that a structure $\mathfrak{A}$ of the vocabulary $\sigma$ is $S$-tilable if and only if there is an expansion $\mathfrak{A}^{*}$ of the structure $\mathfrak{A}$ to the vocabulary $\sigma \cup S$ such that $\mathfrak{A}^{*}=\varphi_{S}$.

Now recall Lemma 4.13. By Lemma 4.13, since $T$ extends linearly in $2 \mathrm{D}$, there exists a computable function $I$ such that for each input $S$ to the tiling problem, the function $I$ is a uniform interpretation of some class $\mathcal{G}(T, S) \supseteq \mathfrak{G}_{S}$ of $S$-labelled supergrids in the class $\mathcal{C}(T, S)$ of all $S$-labelled Cartesian frames with the domain $T$.

Let $S$ be an input to the tiling problem. Define the $\tau$-sentence

$$
\psi_{S}:=\varphi_{C f}^{S} \wedge I\left(\varphi_{S}\right) .
$$

We will prove that for each input $S$ to the tiling problem, the following conditions are equivalent.

(1) There exists an expansion $\mathfrak{B}$ of $(T, \beta)$ to the vocabulary $\tau$ such that $\mathfrak{B} \models \psi_{S}$.

(2) The grid $\mathfrak{G}$ is $S$-tilable.

Thereby we establish that there exists a computable reduction from the complement problem of the tiling problem to the membership problem of the first-order theory of the unary expansion class of $(T, \beta)$. Since the tiling problem is $\Pi_{1}^{0}$-complete, its complement problem is $\Sigma_{1}^{0}$-complete. ${ }^{1}$

Let $S$ be an input to the tiling problem. Assume first that the grid $\mathfrak{G}$ is $S$-tilable. Therefore there exists an expansion $\mathfrak{G}^{*}$ of the grid $\mathfrak{G}$ to the vocabulary $\{H, V\} \cup S$ such that $\mathfrak{G}^{*} \models \varphi_{S}$. Now since $\mathfrak{G}^{*} \in \mathfrak{G}_{S} \subseteq \mathcal{G}(T, S)$, by Lemma 2.1 there exists an $S$-labelled Cartesian frame $\mathfrak{C}$ with the domain $T$ such that $\mathfrak{C} \models I\left(\varphi_{S}\right)$. Since $\mathfrak{C}$ is an $S$-labelled Cartesian frame, we have $\mathfrak{C} \models \varphi_{C f}^{S}$. Therefore $\mathfrak{C}=\varphi_{C f}^{S} \wedge I\left(\varphi_{S}\right)$. Hence the Cartesian frame $\mathfrak{C}$ is an expansion of $(T, \beta)$ such that $\mathfrak{C}=\psi_{S}$.

For the converse, assume that there exists an expansion $\mathfrak{B}$ of $(T, \beta)$ to the vocabulary $\tau$ such that we have $\mathfrak{B} \models \psi_{S}$. Therefore $\mathfrak{B} \models \varphi_{C f}^{S}$ and $\mathfrak{B} \models I\left(\varphi_{S}\right)$. Since $\mathfrak{B} \models \varphi_{C f}^{S}$, the structure $\mathfrak{B}$ is an $S$-labelled Cartesian frame with the domain $T$. Therefore, and since $\mathfrak{B} \models I\left(\varphi_{S}\right)$, we conclude by Lemma 2.1 that $\mathfrak{A} \models \varphi_{S}$ for some $S$-labelled supergrid $\mathfrak{A} \in \mathcal{G}(T, S)$. Thus there exists a supergrid that is $S$-tilable. Hence the grid $\mathfrak{G}$ is $S$-tilable.

As a partial converse to Theorem 4.15, we note that $T$ extending linearly $1 \mathrm{D}$ is not a sufficient condition for undecidability of even the monadic $\Pi_{1}^{1}$-theory of $(T, \beta)$. For instance, the monadic $\Pi_{1}^{1}$-theory of $(\mathbb{R}, \beta)$ is decidable; this follows trivially from the known result that the monadic $\Pi_{1}^{1}$-theory $(\mathbb{R}, \leq)$ is decidable, see $[9]$. Also the monadic $\Pi_{1}^{1}$-theory of $(\mathbb{Q}, \beta)$ is decidable since the MSO-theory of $(\mathbb{Q}, \leq)$ is decidable [25].

\footnotetext{
${ }^{1}$ It is of course a well-known triviality that the complement $\bar{A}$ of a problem $A$ is $\Sigma_{1}^{0}$-hard if $A$ is $\Pi_{1}^{0}$-hard. Choose an arbitrary problem $B \in \Sigma_{1}^{0}$. Therefore $\bar{B} \in \Pi_{1}^{0}$. By the hardness of $A$, there is a computable reduction $f$ such that $x \in \bar{B} \Leftrightarrow f(x) \in A$, whence $x \in B \Leftrightarrow f(x) \in \bar{A}$.
} 
Theorem 4.16. Let $n \geq 2$ be an integer. The first-order theory of the unary expansion class of $\left(\mathbb{R}^{n}, \beta\right)$ is $\Pi_{1}^{1}$-hard.

Proof. The proof is essentially the same as the proof of Theorem 4.15. The main difference is that we use Lemma 4.14 and interpret a class of labelled recurrence grids instead of a class of labelled supergrids, and hence obtain a reduction from the recurrent tiling problem instead of the ordinary tiling problem. Thereby we establish $\Pi_{1}^{1}$-hardness instead of $\Sigma_{1}^{0}$-hardness. Due to the recurrence condition of the recurrent tiling problem, the result of Lemma 4.7 that there is an isomorphism from $(\mathbb{N}$, succ $)$ to $(P, E)$-rather than an embedding - is essential.

Corollary 4.17. Let $n \geq 2$ be an integer. The monadic $\Pi_{1}^{1}$-theory of $\left(\mathbb{R}^{n}, \beta\right)$ is not arithmetical.

\section{Geometric structures $(T, \beta)$ With an undecidable expansion Class With a FINITE UNARY PREDICATE}

In this section we establish undecidability of the first-order theory of the expansion class

$$
\{(T, \beta, P) \mid P \subseteq T \text { is finite }\}
$$

of any geometric structure $(T, \beta)$ such that $T$ extends linearly in $2 \mathrm{D}$. More precisely, we show that any such theory is $\Pi_{1}^{0}$-hard. We prove this by a reduction from the periodic tiling problem to the problem of deciding satisfiability of $\left\{\beta, P, p_{0}, p_{x} . p_{y}\right\}$-sentences in the class of expansions of $(T, \beta)$ by a finite unary predicate $P$ and constants $p_{0}, p_{x}, p_{y}$. The argument is based on interpreting tori in $(T, \beta)$. Most notions used in this section are inherited either directly or with minor adjustments from Section 4

Let $Q$ be a subset of $T \subseteq \mathbb{R}^{n}$. We say that $Q$ is a finite sequence in $T$ if $Q$ is a finite nonempty set and the points in $Q$ are collinear.

Definition 5.1. Let $T \subseteq \mathbb{R}^{n}$ and let $\beta$ be the corresponding betweenness relation. Let $P \subseteq T$ be a finite set, and let $p_{0}, p_{x}, p_{y} \in P$. We call the structure

$$
\mathfrak{C}=\left(T, \beta, P, p_{0}, p_{x}, p_{y}\right)
$$

a finite Cartesian frame with domain $T$ if the following conditions hold.

(1) The points $p_{0}, p_{x}$ and $p_{y}$ are not collinear.

(2) For each point $p \in P$ and $q \in P$ such that $\beta^{*}\left(p_{0}, p, p_{x}\right)$ and $\beta^{*}\left(p_{0}, q, p_{y}\right)$ hold in $\mathfrak{C}$, the unique lines $L_{p}$ and $L_{q}$ in $T$ such that $p, p_{y} \in L_{p}$ and $q, p_{x} \in L_{q}$, intersect. In other words, there exists a point $u \in T$ that lies on both lines $L_{p}$ and $L_{q}$.

If $m$ and $k$ are positive integers such that

$$
\begin{aligned}
& \mid\left\{u \in P \mid \beta\left(p_{0}, u, p_{x}\right) \text { holds in } \mathfrak{C}\right\} \mid=m+2 \text { and } \\
& \mid\left\{u \in P \mid \beta\left(p_{0}, u, p_{y}\right) \text { holds in } \mathfrak{C}\right\} \mid=k+2,
\end{aligned}
$$

we call $\left(T, \beta, P, p_{0}, p_{x}, p_{y}\right)$ an $m \times k$ Cartesian frame with domain $T$.

Definition 5.2. Let $\mathfrak{C}=\left(T, \beta, P, p_{0}, p_{x}, p_{y}\right)$ be a finite Cartesian frame. Let $p, q \in P$, $p \neq p_{x}, q \neq p_{y}$, be points such that $\beta\left(p_{0}, p, p_{x}\right)$ and $\beta\left(p_{0}, q, p_{y}\right)$ hold in $\mathfrak{C}$. Let $L_{p}$ and $L_{q}$ be the lines in $T$ such that $p, p_{y} \in L_{p}$ and $q, p_{x} \in L_{q}$. The point $u \in T$ that lies on both lines $L_{p}$ and $L_{q}$ is called the intersection point of $\mathfrak{C}$ corresponding to the pair $(p, q)$. A point $u \in T$ is called an intersection point of the finite Cartesian frame $\mathfrak{C}$, if it is an intersection point of $\mathfrak{C}$ corresponding to some pair $(p, q)$. 
Definition 5.3. Let $\mathfrak{C}=\left(T, \beta, P, p_{0}, p_{x}, p_{y}\right)$ be a finite Cartesian frame. Let $p, p^{\prime}, q, q^{\prime} \in P$ be points such that the following conditions hold.

(1) $\beta\left(p_{0}, p, p^{\prime}\right)$ and $\beta^{*}\left(p, p^{\prime}, p_{x}\right)$ hold in $\mathfrak{C}$.

(2) $\beta\left(p_{0}, q, q^{\prime}\right)$ and $\beta^{*}\left(q, q^{\prime}, p_{y}\right)$ hold in $\mathfrak{C}$.

(3) There does not exist a point $u \in P$ such that $\beta^{*}\left(p, u, p^{\prime}\right)$ or $\beta^{*}\left(q, u, q^{\prime}\right)$ holds in $\mathfrak{C}$.

Let $u$ be the intersection point of $\mathfrak{C}$ corresponding to $(p, q)$ and $v$ the intersection point of $\mathfrak{C}$ correponding to $\left(p^{\prime}, q^{\prime}\right)$. We say that $v$ is the diagonal successor of $u$ in $\mathfrak{C}$.

Definition 5.4. Let $\mathfrak{C}=\left(T, \beta, P, p_{0}, p_{x}, p_{y}\right)$ be a finite Cartesian frame and let $S$ be a finite nonempty set of tile symbols. We call $\mathfrak{C}$ an $S$-labelled finite Cartesian frame if the number of points in $P$ strictly between any intersection point $u$ of $\mathfrak{C}$ and its diagonal successor $v$ is in the set $\left\{N\left(P_{t}\right) \mid P_{t} \in S\right\}$. We let $\mathcal{C}^{\text {fin }}(T, S)$ denote the class of $S$-labelled finite Cartesian frames with domain $T$.

Lemma 5.5. Let $T \subseteq \mathbb{R}^{n}, n \geq 2$. Let $\mathcal{C}$ be the class of all expansions $\left(T, \beta, P, p_{0}, p_{x}, p_{y}\right)$ of $(T, \beta)$ by a finite unary relation $P$ and constants $p_{0}, p_{x}$ and $p_{y}$. There is a computable function associating each finite nonempty set of tile symbols $S$ with a first-order $\left\{\beta, P, p_{0}, p_{x}, p_{y}\right\}$ sentence $\varphi_{\text {fCf }}^{S}$ that defines the class $\mathcal{C}^{\text {fin }}(T, S)$ with respect to the class $\mathcal{C}$.

Proof. Straightforward.

Let $S \neq \emptyset$ be a finite set of tile symbols. Let $\mathcal{T}_{S}$ denote the class of structures $\mathfrak{A}$ that satisfy the following conditions.

(1) The structure $\mathfrak{A}$ is an expansion of some torus to the vocabulary $\{V, H\} \cup S$.

(2) Each point in the domain of $\mathfrak{A}$ is in the extension of exactly one predicate symbol $P_{t} \in S$. Structures in the class $\mathcal{T}_{S}$ are called $S$-labelled tori.

Lemma 5.6. Let $T \subseteq \mathbb{R}^{n}, n \geq 2$. Assume that $T$ extends linearly in 2D. There is a computable function $J$ such that for all finite sets $S \neq \emptyset$ of tile symbols, $J$ is a uniform interpretation of the class of $S$-labelled tori in $\mathcal{C}^{\text {Fin }}(T, S)$.

Proof. Let $S$ be a finite nonempty set of tile symbols. Let $\mathfrak{C}=\left(T, \beta, P, p_{0}, p_{x}, p_{y}\right)$ be an $S$-labelled $m \times k$ Cartesian frame. We shall show how to interpret an $S$-labelled $m \times k$ torus $\mathfrak{T}_{\mathfrak{C}}$ in $\mathfrak{C}$. The idea behind the interpretation is quite similar to the idea behind the interpretation in the proof of Lemma 4.13 .

Recall the formulae $\varphi_{D o m}, \varphi_{H}, \varphi_{V}$ and $\varphi_{P_{t}}$ defined in the proof of Lemma 4.13. We shall now define variants of these formulae suitable for interpreting $S$-labelled tori in $S$-labelled finite Cartesian frames. In the definitions of the new formulae, we shall make use of the old formulae $\varphi_{D o m}, \varphi_{H}, \varphi_{V}$ and $\varphi_{P_{t}}$.

We define

$$
\begin{aligned}
\varphi_{D o m}^{f i n}(u) & :=\varphi_{D o m}(u) \wedge \exists x \exists y\left(\varphi_{D o m}(x) \wedge \varphi_{D o m}(y) \wedge \varphi_{H}(u, x) \wedge \varphi_{V}(u, y)\right), \\
\varphi_{H}^{f i n}(u, v) & :=\varphi_{H}(u, v) \vee\left(\beta\left(p_{0}, v, p_{y}\right) \wedge \beta\left(v, u, p_{x}\right) \wedge \forall x\left(\beta^{*}\left(u, x, p_{x}\right) \rightarrow \neg \varphi_{D o m}^{f i n}(x)\right)\right), \\
\varphi_{V}^{f i n}(u, v) & :=\varphi_{V}(u, v) \vee\left(\beta\left(p_{0}, v, p_{x}\right) \wedge \beta\left(v, u, p_{y}\right) \wedge \forall x\left(\beta^{*}\left(u, x, p_{y}\right) \rightarrow \neg \varphi_{D o m}^{f i n}(x)\right)\right), \\
\varphi_{P_{t}}^{f i n}(u) & :=\varphi_{P_{t}}(u) \text { for each } P_{t} \in S .
\end{aligned}
$$

Let $F_{\mathfrak{C}}:=\left\{u \in T \mid \mathfrak{C} \models \varphi_{\text {Dom }}^{\text {fin }}(u)\right\}$. Define the structure

$$
\mathfrak{F}_{\mathfrak{C}}=\left(F_{\mathfrak{C}}, H^{\mathfrak{F}_{\mathfrak{C}}}, V^{\mathfrak{F}_{\mathfrak{C}}},\left(P_{t}^{\mathfrak{F}_{\mathfrak{C}}}\right)_{P_{t} \in S}\right),
$$


where

$$
\begin{aligned}
H^{\mathfrak{F}_{\mathfrak{C}}} & :=\left\{(u, v) \in F_{\mathfrak{C}} \times F_{\mathfrak{C}} \mid \mathfrak{C}=\varphi_{H}^{f i n}(u, v)\right\}, \\
V^{\mathfrak{F}_{\mathfrak{C}}} & :=\left\{(u, v) \in F_{\mathfrak{C}} \times F_{\mathfrak{C}} \mid \mathfrak{C}=\varphi_{V}^{f i n}(u, v)\right\}, \\
P_{t}^{\mathfrak{F}_{\mathfrak{C}}} & :=\left\{u \in F_{\mathfrak{C}} \mid \mathfrak{C}=\varphi_{P_{t}}^{f i n}(u)\right\},
\end{aligned}
$$

for all $P_{t} \in S$. It is straightforward to check that there exists an $S$-labelled $m \times k$ torus

$$
\mathfrak{T}_{\mathfrak{C}}=\left(D, H^{\mathfrak{T}_{\mathfrak{C}}}, V^{\mathfrak{T}_{\mathfrak{C}}},\left(P_{t}^{\mathfrak{T}_{\mathfrak{C}}}\right)_{P_{t} \in S}\right)
$$

and a bijection $f$ from $D$ to $F_{\mathfrak{C}}$ such that the following conditions hold for all $u, v \in D$.

(1) $(u, v) \in H^{\mathfrak{T}_{\mathfrak{C}}} \Leftrightarrow \varphi_{H}^{f i n}(f(u), f(v))$,

(2) $(u, v) \in V^{\mathfrak{T}_{\mathfrak{C}}} \Leftrightarrow \varphi_{V}^{f i n}(f(u), f(v))$,

(3) $u \in P_{t}^{\mathfrak{T}_{\mathfrak{C}}} \Leftrightarrow \varphi_{P_{t}}^{f i n}(f(u))$ for all $P_{t} \in S$.

Notice that since $T$ extends linearly in $2 \mathrm{D}$, there exist $S$-labelled finite Cartesian frames of all sizes $m \times k$ with all possible $S$-labelling configurations in the class $\mathcal{C}^{f i n}(T, S)$. We have hence established that for all finite sets $S \neq \emptyset$ of tile symbols, the class of $S$-labelled tori is uniformly first-order interpretable in the class of $S$-labelled finite Cartesian frames with the domain $T$. Furthermore, the formulae $\varphi_{D o m}^{f i n}, \varphi_{H}^{f i n}, \varphi_{V}^{f i n}$ and $\varphi_{P_{t}}^{f i n}$ define the desired uniform interpretation $J$.

Theorem 5.7. Let $T \subseteq \mathbb{R}^{n}$ and let $\beta$ be the corresponding betweenness relation. Assume that $T$ extends linearly in 2D. The first-order theory of the class $\{(T, \beta, P) \mid P \subseteq T$ is finite $\}$ is $\Pi_{1}^{0}$-hard.

Proof. Since $T$ extends linearly in 2D, we have $n \geq 2$. Let $\sigma=\{H, V\}$ be the vocabulary of tori, and let $\tau=\left\{\beta, P, p_{0}, p_{x}, p_{y}\right\}$ be the vocabulary of labelled finite Cartesian frames. By Lemma 5.5, there is a computable function that associates each input $S$ to the periodic tiling problem with a first-order $\tau$-sentence that defines the class of $S$-labelled finite Cartesian frames with the domain $T$ with respect to the class of all expansions of $(T, \beta)$ to the vocabulary $\tau$. Let $\varphi_{f C f}^{S}$ denote such a sentence. By Lemma 5.6, there is a computable function $J$ such that for all inputs $S$ to the periodic tiling problem, the function $J$ is a uniform interpretation of the class of $S$-labelled tori in the class of $S$-labelled finite Cartesian frames with domain $T$. By Lemma 2.5, there is a computable function that associates each input $S$ to the periodic tiling problem with a first-order $\sigma \cup S$-sentence $\varphi_{S}$ such that for all tori $\mathfrak{B}$, the torus $\mathfrak{B}$ is $S$-tilable iff there is an expansion $\mathfrak{B}^{*}$ of $\mathfrak{B}$ to the vocabulary $\sigma \cup S$ such that $\mathfrak{B}^{*}=\varphi_{S}$.

Let $S$ be a finite nonempty set of tile symbols. Define the first-order $\tau$-sentence

$$
\gamma_{S}:=\varphi_{f C f}^{S} \wedge J\left(\varphi_{S}\right)
$$

We will prove that for each input $S$ to the periodic tiling problem, the following conditions are equivalent.

(1) There exists an expansion $\mathfrak{B}=\left(T, \beta, P, p_{0}, p_{x}, p_{y}\right)$ of $(T, \beta)$ by a finite unary relation $P \subseteq T$ and constants $p_{0}, p_{x}, p_{y} \in T$ such that $\mathfrak{B}=\gamma_{S}$.

(2) There exists a torus $\mathfrak{T}$ such that $\mathfrak{T}$ is $S$-tilable.

Thereby we establish that there exists a computable reduction from the complement problem of the periodic tiling problem to the membership problem of the first-order theory of the 
unary expansion class of $(T, \beta)$ with a finite predicate. Since the periodic tiling problem is $\Sigma_{1}^{0}$-complete, its complement problem is $\Pi_{1}^{0}$-complete.

Let $S$ be an input to the periodic tiling problem. First assume that there exists a torus $\mathfrak{T}$ such that $\mathfrak{T}$ is $S$-tilable. Therefore, by Lemma 2.5, there exists an expansion $\mathfrak{T}^{*}$ of $\mathfrak{T}$ to the vocabulary $\sigma \cup S$ such that $\mathfrak{T}^{*}=\varphi_{S}$. Since the function $J$ is a uniform interpretation of the class of $S$-labelled tori in the class of $S$-labelled finite Cartesian frames with the domain $T$, and since $\mathfrak{T}^{*}=\varphi_{S}$, it follows by Lemma 2.1 that there exists an $S$-labelled finite Cartesian frame $\mathfrak{C}$ with the domain $T$ such that $\mathfrak{C}=J\left(\varphi_{S}\right)$. Since $\mathfrak{C}$ is an $S$-labelled finite Cartesian frame, we have that $\mathfrak{C}=\varphi_{f c f}^{S}$. Therefore $\mathfrak{C}=\varphi_{f c f}^{S} \wedge J\left(\varphi_{S}\right)$. Hence the finite Cartesian frame $\mathfrak{C}$ is an expansion of $(T, \beta)$ by a finite unary relation $P \subseteq T$ and constants $p_{0}, p_{x}, p_{y} \in T$ such that $\mathfrak{C}=\gamma_{S}$.

For the converse, assume that there exists an expansion $\mathfrak{B}=\left(T, \beta, P, p_{0}, p_{x}, p_{y}\right)$ of $(T, \beta)$ by a finite unary relation $P \subseteq T$ and constants $p_{0}, p_{x}, p_{y} \in T$ such that $\mathfrak{B} \mid=\gamma_{S}$. Therefore $\mathfrak{B} \models \varphi_{f C f}^{S}$ and $\mathfrak{B} \models J_{S}\left(\varphi_{S}\right)$. Since $\mathfrak{B} \models \varphi_{f C f}^{S}$, the structure $\mathfrak{B}$ is an $S$-labelled finite Cartesian frame with domain $T$. Therefore, and since $\mathfrak{B} \models J\left(\varphi_{S}\right)$, we conclude by Lemma 2.1 that $\mathfrak{A}=\varphi_{S}$ holds for some $S$-labelled torus $\mathfrak{A}$. Hence by Lemma 2.5 there exists a torus that is $S$-tilable.

\section{Conclusions}

We have studied first-order theories of unary expansion classes of geometric structures $(T, \beta)$, $T \subseteq \mathbb{R}^{n}$. We have established that for $n \geq 2$, the first-order theory of the class of all expansions of $\left(\mathbb{R}^{n}, \beta\right)$ with a single unary predicate is highly undecidable $\left(\Pi_{1}^{1}\right.$-hard). This refutes a conjecture from the article [3] of Aiello and van Benthem. In addition, we have established the following for any geometric structure $(T, \beta)$ that extends linearly in 2D.

(1) The first-order theory of the class of expansions of $(T, \beta)$ with a single unary predicate is $\Sigma_{1}^{0}$-hard.

(2) The first-order theory of the class of expansions of $(T, \beta)$ with a single finite unary predicate is $\Pi_{1}^{0}$-hard.

Geometric structures that extend linearly in 2D include, for example, the rational plane $\left(\mathbb{Q}^{2}, \beta\right)$ and the real unit rectangle $\left([0,1]^{2}, \beta\right)$, to name a few.

The techniques used in the proofs can be easily modified to yield undecidability of first-order theories of a significant variety of natural restricted expansion classes of the affine real plane $\left(\mathbb{R}^{2}, \beta\right)$, such as those with a unary predicate denoting a polygon, a finite union of closed rectangles, and a semialgebraic set, for example. Such classes could be interesting from the point of view of applications.

In addition to studying issues of decidability, we briefly compared the expressivities of universal monadic second-order logic and weak universal monadic second-order logic. While the two are incomparable in general, we established that over any class of expansions of $\left(\mathbb{R}^{n}, \beta\right)$, it is no longer the case. We showed that finiteness of a unary predicate is definable by a first-order sentence, and hence obtained translations from $\forall$ WMSO into $\forall \mathrm{MSO}$ and from WMSO into MSO.

Our original objective to study expansion classes of $\left(\mathbb{R}^{n}, \beta\right)$ was to identify decidable logics of space with distinguished regions. Due to the ubiquitous applicability of the tiling methods, this pursuit gave way to identifying several undecidable theories of geometry. Hence we shall look elsewhere in order to identify well behaved natural decidable logics of 
space. Possible interesting directions include considering natural fragments of first-order logic over expansions of $\left(\mathbb{R}^{n}, \beta\right)$, and also other geometries. Related results could provide insight, for example, in the background theory of modal spatial logics.

\section{REFERENCES}

[1] Marco Aiello, Ian Pratt-Hartmann, and Johan Benthem. What is spatial logic? In Marco Aiello, Ian Pratt-Hartmann, and Johan van Benthem, editors, Handbook of Spatial Logics, pages 1-11. Springer Netherlands, 2007.

[2] Marco Aiello, Ian Pratt-Hartmann, and Johan van Benthem, editors. Handbook of Spatial Logics. Springer, 2007.

[3] Marco Aiello and Johan van Benthem. A modal walk through space. Journal of Applied Non-classical Logics, 12:319-363, 2002.

[4] Philippe Balbiani, Luis Farias del Cerro, Tinko Tinchev, and Dimiter Vakarelov. Modal logics for incidence geometries. Journal of Logic and Computation, 7(1):59-78, 1997.

[5] Philippe Balbiani and Valentin Goranko. Modal logics for parallelism, orthogonality, and affine geometries. Journal of Applied Non-Classical Logics, 12(3-4):365-398, 2002.

[6] Philippe Balbiani, Valentin Goranko, Ruaan Kellerman, and Dimiter Vakarelov. Logical theories for fragments of elementary geometry. In Marco Aiello, Ian Pratt-Hartmann, and Johan van Benthem, editors, Handbook of Spatial Logics, pages 343-428. Springer, 2007.

[7] Robert Berger. The Undecidability of the Domino Problem. American Mathematical Society memoirs. American Mathematical Society, 1966.

[8] Jacek Bochnak, Michel Coste, and Marie-Francoise Roy. Real Algebraic Geometry. Springer, 1998.

[9] John P. Burgess and Yuri Gurevich. The decision problem for linear temporal logic. Notre Dame Journal of Formal Logic, 26(2):115-128, April 1985.

[10] Balder ten Cate and Alessandro Facchini. Characterizing EF over infinite trees and modal logic on transitive graphs. In Filip Murlak and Piotr Sankowski, editors, MFCS, volume 6907 of Lecture Notes in Computer Science, pages 290-302. Springer, 2011.

[11] Aled Griffiths. Computational Properties of Spatial Logics in the Real Plane. PhD thesis, University of Manchester, 2008.

[12] Yuri Gurevich. Monadic second-order theories. In Jon Barwise and Solomon Feferman, editors, ModelTheoretic Logics, pages 479-506. Springer, New York, 1985.

[13] Yuri Gurevich and Igor O. Koryakov. Remarks on berger's paper on the domino problem. Siberian Mathematical Journal, 13:319-321, 1972.

[14] Marc Gyssens, Jan Van den Bussche, and Dirk Van Gucht. Complete geometric query languages. Journal of Computer and System Sciences, 58(3):483-511, 1999.

[15] David Harel. Recurring dominoes: Making the highly undecidable highly understandable. Annals of Discrete Mathematics, 24:51-72, 1985.

[16] Ian Hodkinson and Altaf Hussain. The modal logic of affine planes is not finitely axiomatisable. Journal of Symbolic Logic, 73(3):940-952, 2008.

[17] Roman Kontchakov, Ian Pratt-Hartmann, Frank Wolter, and Michael Zakharyaschev. Spatial logics with connectedness predicates. Logical Methods in Computer Science, 6(3), 2010.

[18] Bart Kuijpers and Jan Van den Bussche. Logical aspects of spatial databases., pages 77-108. Cambridge: Cambridge University Press, 2011.

[19] Antti Kuusisto, Jeremy Meyers, and Jonni Virtema. Undecidable First-Order Theories of Affine Geometries. In Patrick Cégielski and Arnaud Durand, editors, Computer Science Logic (CSL'12) - 26th International Workshop/21st Annual Conference of the EACSL, volume 16 of Leibniz International Proceedings in Informatics (LIPIcs), pages 470-484, Dagstuhl, Germany, 2012. Schloss Dagstuhl-LeibnizZentrum fuer Informatik.

[20] Leonid Libkin. Elements of Finite Model Theory. Springer, 2004.

[21] Yavor Nenov and Ian Pratt-Hartmann. On the computability of region-based euclidean logics. In Anuj Dawar and Helmut Veith, editors, CSL, volume 6247 of Lecture Notes in Computer Science, pages 439-453. Springer, 2010. 
[22] Victor Pambuccian. The axiomatics of ordered geometry: I. ordered incidence spaces. Expositiones Mathematicae, 29(1):24 - 66, 2011.

[23] Alexander Prestel. Zur Axiomatisierung gewisser affiner Geometrien. L'Enseignement Mathématique (2), 27:125-136, 1981.

[24] Alexander Prestel and Leslaw W. Szczerba. Nonaxiomatizability of real general affine geometry. Fundamenta Mathematicae, 104:193-202, 1979.

[25] Michael O. Rabin. Decidability of Second-order Theories and Automata on Infinite Trees. IBM Watson Research Center, 1968.

[26] Mikhail Sheremet, Frank Wolter, and Michael Zakharyaschev. A modal logic framework for reasoning about comparative distances and topology. Annals of Pure and Applied Logic, 161(4):534-559, 2010.

[27] Wolfram Schwabhäuser; Wanda Szmielew and Alfred Tarski. Metamathematical methods in geometry. Part I: An axiomatic building of Euclidean geometries. Part II: Metamathematical considerations. With a new foreword by Michael Beeson. (Metamathematische Methoden in der Geometrie. Teil I: Ein axiomatischer Aufbau der euklidischen Geometrie. Teil II: Metamathematische Betrachtungen.) Reprint of the 1983 original published by Springer. Bronx, NY: Ishi Press International, reprint of the 1983 original published by springer edition, 2011.

[28] Alfred Tarski. A decision method for elementary algebra and geometry. University of California Press, Berkeley, 5, 1951.

[29] Alfred Tarski and Steven Givant. Tarski's system of geometry. The Bulletin of Symbolic Logic, 5(2):175214, 1999.

[30] Tinko Tinchev and Dimiter Vakarelov. Logics of space with connectedness predicates: Complete axiomatizations. In Lev D. Beklemishev, Valentin Goranko, and Valentin Shehtman, editors, Advances in Modal Logic, pages 434-453. College Publications, 2010.

[31] Yde Venema. Points, lines and diamonds: A two-sorted modal logic for projective planes. Journal of Logic and Computation, 9(5):601-621, 1999. 